H. Lang, M. Arnold

Numerical aspects in the dynamic simulation of geometrically exact rods 
(C) Fraunhofer-Institut für Techno- und Wirtschaftsmathematik ITWM 2009

ISSN 1434-9973

Bericht 179 (2009)

Alle Rechte vorbehalten. Ohne ausdrückliche schriftliche Genehmigung des Herausgebers ist es nicht gestattet, das Buch oder Teile daraus in irgendeiner Form durch Fotokopie, Mikrofilm oder andere Verfahren zu reproduzieren oder in eine für Maschinen, insbesondere Datenverarbeitungsanlagen, verwendbare Sprache zu übertragen. Dasselbe gilt für das Recht der öffentlichen Wiedergabe.

Warennamen werden ohne Gewährleistung der freien Verwendbarkeit benutzt.

Die Veröffentlichungen in der Berichtsreihe des Fraunhofer ITWM können bezogen werden über:

Fraunhofer-Institut für Techno- und Wirtschaftsmathematik ITWM Fraunhofer-Platz 1

67663 Kaiserslautern

Germany

Telefon: $\quad+49(0) 631 / 31600-0$

Telefax: $\quad+49(0) 631 / 31600-1099$

E-Mail: info@itwm.fraunhofer.de

Internet: www.itwm.fraunhofer.de 
Das Tätigkeitsfeld des Fraunhofer-Instituts für Techno- und Wirtschaftsmathematik ITWM umfasst anwendungsnahe Grundlagenforschung, angewandte Forschung sowie Beratung und kundenspezifische Lösungen auf allen Gebieten, die für Techno- und Wirtschaftsmathematik bedeutsam sind.

In der Reihe »Berichte des Fraunhofer ITWM « soll die Arbeit des Instituts kontinuierlich einer interessierten Öffentlichkeit in Industrie, Wirtschaft und Wissenschaft vorgestellt werden. Durch die enge Verzahnung mit dem Fachbereich Mathematik der Universität Kaiserslautern sowie durch zahlreiche Kooperationen mit internationalen Institutionen und Hochschulen in den Bereichen Ausbildung und Forschung ist ein großes Potenzial für Forschungsberichte vorhanden. In die Berichtreihe werden sowohl hervorragende Diplom- und Projektarbeiten und Dissertationen als auch Forschungsberichte der Institutsmitarbeiter und Institutsgäste zu aktuellen Fragen der Techno- und Wirtschaftsmathematik aufgenommen.

Darüber hinaus bietet die Reihe ein Forum für die Berichterstattung über die zahlreichen Kooperationsprojekte des Instituts mit Partnern aus Industrie und Wirtschaft.

Berichterstattung heißt hier Dokumentation des Transfers aktueller Ergebnisse aus mathematischer Forschungs- und Entwicklungsarbeit in industrielle Anwendungen und Softwareprodukte - und umgekehrt, denn Probleme der Praxis generieren neue interessante mathematische Fragestellungen.

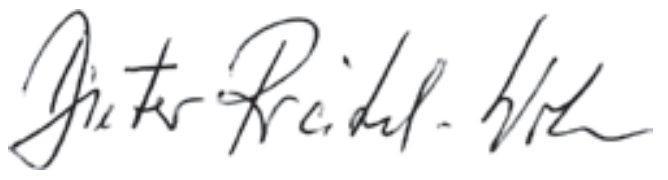

Prof. Dr. Dieter Prätzel-Wolters Institutsleiter

Kaiserslautern, im Juni 2001 



\title{
Numerical aspects in the dynamic simulation of geometrically exact rods
}

\author{
Holger LANG ${ }^{b}$, Martin ARnOLD \\ ${ }^{b}$ Fraunhofer Institute for Industrial Mathematics \\ Fraunhofer Platz 1, 67663 Kaiserslautern, Germany \\ holger.lang@itwm.fraunhofer.de, \\ \# Institute for Mathematics, Martin-Luther-Universität Halle-Wittenberg \\ 06099 Halle (Saale), Germany \\ martin.arnold@mathematik.uni-halle.de
}

\begin{abstract}
Classical geometrically exact Kirchhoff and Cosserat models are used to study the nonlinear deformation of rods. Extension, bending and torsion of the rod may be represented by the Kirchhoff model. The Cosserat model additionally takes into account shearing effects. Second order finite differences on a staggered grid define discrete viscoelastic versions of these classical models. Since the rotations are parametrised by unit quaternions, the space discretisation results in differential-algebraic equations that are solved numerically by standard techniques like index reduction and projection methods. Using absolute coordinates, the mass and constraint matrices are sparse and this sparsity may be exploited to speed-up time integration. Further improvements are possible in the Cosserat model, because the constraints are just the normalisation conditions for unit quaternions such that the null space of the constraint matrix can be given analytically. The results of the theoretical investigations are illustrated by numerical tests.
\end{abstract}

Keywords. Kirchhoff and Cosserat rods, Geometrically exact rods, Deformable bodies, Multibody dynamics, Partial differential algebraic equations, Method of lines, Time integration.

MSC Classification: 35Q74, 65L04, 65L12, 65L80, 74K10.

\section{Introduction}

Rod models are a classical subject of mechanics with numerous applications in engineering. Nevertheless, the dynamical analysis of fully nonlinear beams and rods in 3D is even today a challenging problem, both from the viewpoint of modeling and from the viewpoint of the efficient numerical solution of the resulting model equations [14, 21, 23, 36, 38, 39]. In the present paper, we combine an objective/frame-indifferent geometrically exact space discretisation of Kirchhoff and Cosserat rods with standard methods for the time integration of the equations of motion for constrained mechanical systems $[4,17,22]$.

Following the method of lines, the equations of motion for Kirchhoff rods and Cosserat rods are discretised first in space by finite differences on a staggered grid [27]. The rotations are parametrised by unit quaternions resulting in constraints to guarantee the normalisation of the quaternions. In the Kirchhoff model, additional constraints result from the inhibition of shearing effects. In a hierarchy of rod models, the inextensible Kirchhoff model is the physically most simple one but results in even more complex constraints than in the case of extensible Kirchhoff rods. On the other hand, the consideration of shearing effects in the Cosserat model introduces very stiff potential forces in the semi-discretised system.

This work was motivated by the need for real-time capable simulation methods for deformable cables, tubes and hoses in industrial applications. Exploiting the specific structure of the model equations, the numerical effort can be reduced by several orders of magnitude in all three nonlinear rod models. In the semi-discretised system, the nonlinear deformation of the rod is described by the Cartesian coordinates $x=x(s, t)$ of its centerline and the corresponding orthonormal frame field that is defined by quaternions $p=p(s, t)$ of unit length $\|p(s, t)\|=1$. It is well known 
from multibody dynamics [36] that such absolute coordinates result in rather large differentialalgebraic systems with sparse mass and constraint matrices. The sparsity pattern is in general determined by the topology of a multibody system [17]. For rod like structures, mass and constraint matrices have band structure and the use of structure exploiting versions of Gaussian elimination is straightforward [19].

Because of the constraints, the semi-discretised system forms a differential-algebraic equation (DAE) of index 3. Differentiating the constraints twice, the analytically equivalent index-1 formulation of the model equations is obtained, which may be solved by any solver for ordinary differential equations (ODEs). The well known drift-off effect is avoided by projection steps that require a small extra effort during time integration [22]. Numerical tests with many standard ODE solvers illustrate the strong stepsize restrictions for explicit solvers resulting from the shear stiffness in the Cosserat model.

On the other hand, the Cosserat model allows a very efficient implementation of the index-2 formulation of the model equations [22]. The constraints in the index-2 formulation are linear w.r.t. the velocities. Because of the very simple structure of the constraints in the quaternionic Cosserat model, the null space of the constraint matrix is given explicitly and the Lagrangian multipliers may be eliminated by a null space method [17]. Recently, this solution technique that combines redundant position coordinates with a (smaller) set of independent velocity coordinates found new interest in multibody dynamics [8]. In numerical tests with implicit and linearly implicit ODE/DAE solvers, the null space approach was clearly superior to the time integration of the index-1 formulation. The drift-off effect was again avoided by projection steps or, alternatively, by Baumgarte stabilisation that gave promising results in the application to semi-discretised stiff Cosserat rods.

We distinguish three basic types of classical rod models. In hierarchical descending order, these are the Cosserat, the extensible Kirchhoff and the inextensible Kirchhoff model [1, 2, 3, 9, $13,14,21,23,24,25,26,27,28,32,38,39]$. Table 1 presents a short overview, including numerical problems in time integration to be discussed in this article.

In section 2, we shortly introduce these three basic classical models in the continuum, where we concentrate on the kinematics - especially kinematic restrictions for the Kirchhoff models -, the strain measures and the internal energies. For the method of lines, in section 3, we summarise a recently introduced new spatial discretisation approach, which is based on geometric finite differences on a staggered grid $[26,27]$. Our ansatz generalises a discrete differential geometric approach in $[7,10]$, where inextensible Kirchhoff rods have been examined. The formulation is appropriate for multibody dynamics, i. e. classical Lagrangian mechanics on manifolds. In section 4, we discuss several numerical topics, which are contained in the lower part of Table 1. All these topics are of importance for a proper and efficient numerical treatment, especially the time integration of the spatially discrete models. Throughout this article, we use absolute coordinate formulation. In addition, we expose and discuss an appropriate null space coordinate formulation for the Cosserat model in section 4 .

\section{Smooth geometrically exact rods}

The Cosserat and Kirchhoff rod models [1, 2, 3, 28, 38, 39] are established for the geometrically exact simulation of deformable slender one dimensional elastic structures (e.g. cables, helicopter or wind turbine rotor blades, steel strings), both statically and dynamically. A Cosserat rod can be considered as the geometrically nonlinear generalisation of a linear Timoshenko-Reissner beam. Likewise, a Kirchhoff rod is a geometrically nonlinear generalisation of a linear Euler-Bernoulli beam. The latter beam models are classical in structural mechanics [13].

In contrast to a Kirchhoff rod, a Cosserat rod allows to model not only bending (= flexure), torsion (= twist) and extension (= dilation), but as well shearing. We subdivide the class of Kirchhoff rod models into two subclasses, which are the extensible (where dilation is allowed) and inextensible Kirchhoff (where dilation is forbidden) models. For all these three basic classical models, the overall deformation as response to external loads, i. e. translations, rotations, forces or moments, may 


\begin{tabular}{|c||c|c|c|}
\hline & COSSERAT & $\begin{array}{c}\text { Extensible } \\
\text { KIRCHHOFF }\end{array}$ & $\begin{array}{c}\text { Inextensible } \\
\text { KIRCHHOFF }\end{array}$ \\
\hline \hline physical DOF & S $(2 \times), \mathrm{E}, \mathrm{B}(2 \times), \mathrm{T}$ & $\mathrm{E}, \mathrm{B}(2 \times), \mathrm{T}$ & $\mathrm{B}(2 \times), \mathrm{T}$ \\
& $\mathbf{6}$ & 3,3 & 3,3 \\
\hline \hline Translations $x, \dot{x}$ & 3,3 & 4,4 & 4,4 \\
\hline Rotations $p, \dot{p}$ & 4,4 & 3 & 4 \\
\hline Lagrange multipliers $\lambda$ & 1 & $\mathbf{1 7}$ & $\mathbf{1 8}$ \\
\hline \hline Total number of unknowns & $\mathbf{1 5}$ & no & yes \\
\hline \hline Kinematic singularities & no & expensive & expensive \\
\hline $\begin{array}{c}\text { Handling of the } \\
\text { mass-constraint matrix }\end{array}$ & cheap & iteratively & iteratively \\
\hline Constraint stabilisation & explicitly & stiff & non-stiff \\
\hline $\begin{array}{c}\text { Numerical stiffness } \\
\text { (for soft materials) }\end{array}$ & highly stiff & & \\
\hline
\end{tabular}

Table 1: The kinematic DOF of the smooth classical rod models and related numerical issues in time integration. (Here $S=$ shearing, $E=$ extension, $B=$ bending, $T=$ torsion.)

become large, although locally the stresses and strains are assumed to remain small. We remark that these rod models serve as kinematic 'skeletons' for the full three dimensional elastic problem. The reconstruction of the three dimensional displacement, stress and strain distributions can be conveniently carried out in a postprocessing by the use of 'warping functions' [15, 21, 28].

In this section, we describe the basic equations in terms of energies for the smooth Cosserat and Kirchhoff rods, where we parametrise the rotatory degrees of freedom directly with quaternions. Unit quaternions in the subgroup $\mathbb{S}^{3}=\partial B_{1}^{\mathbb{H}}(0)=\left\{p \in \mathbb{H}:\|p\|^{2}=1\right\} \subset \mathbb{H}$ are an appropriate way to describe (non-commutative spatial) rotations in $S O(3)=\left\{Q \in \mathbb{R}^{3 \times 3}: Q Q^{\top}=Q^{\top} Q=\right.$ $I, \operatorname{det} Q=1\}$. This is analogous to unit complex numbers in the subgroup $\mathbb{S}^{1}=\partial B_{1}^{\mathbb{C}}(0)=\{z \in \mathbb{C}$ : $\left.\|z\|^{2}=1\right\} \subset \mathbb{C}$, which describe (commutative plane) rotations in $S O(2)=\left\{Q \in \mathbb{R}^{2 \times 2}: Q Q^{\top}=\right.$ $\left.Q^{\top} Q=I, \operatorname{det} Q=1\right\}$. We do not consider other possibilities, such as Rodriguez parameters, rotation vectors, Euler or Cardan angles [11, 17, 34]. All of them have their pros and cons. So as a pro, which in our opinion is the most important one, gimbal locking can be avoided by the use of quaternions. A con is that they must be kept at unit length, which leads to kinematic constraints and DAEs in any case. (But we will see that this is actually not a severe drawback.)

Our starting point for the continuous rod models is the exposition in [3, 38, 39]. For the constitutive material behaviour, we choose a simple linear viscoelastic one that is called "viscoelastic of complexity one' in $[1,2,3]$. The elastic parameters can be straightforwardly deduced from material and geometric ones [28, 39]. Concerning the damping model, we note that it is macroscopic and phenomenological, it comprises not only pure material damping, but also miscellaneous damping mechanisms. We assume both the elastic and viscoelastic parts as diagonal and positive definite. The generalisation to non-diagonal, symmetric and positive definite constitutive Hookean-like tensors or to nonlinear hyperelastic materials is straightforward and does not cause any harm in principle. We concentrate on the description of the internal potential, dissipative and kinetic energies, as these will be the basis for the discrete model later.

We start with the kinematics for the Cosserat model, which is the most general one in the hierarchy. The Kirchhoff models are special cases with kinematic restrictions. A Cosserat rod is completely determined by its centerline of mass centroids

$$
x:[0, L] \times[0, T] \rightarrow \mathbb{R}^{3}, \quad(s, t) \mapsto x(s, t)
$$

and its unit quaternion field

$$
p:[0, L] \times[0, T] \rightarrow \mathbb{S}^{3}=\partial B_{1}^{\mathbb{H}}(0) \hookrightarrow \mathbb{H}, \quad(s, t) \mapsto p(s, t) .
$$


The quaternion field uniquely determines its orthonormal frame field

$$
\Lambda \circ p:[0, L] \times[0, T] \stackrel{p}{\longrightarrow} \mathbb{S}^{3}=\partial B_{1}^{\mathbb{H}}(0) \stackrel{\Lambda}{\longrightarrow} S O(3), \quad(s, t) \mapsto \Lambda(p(s, t))
$$

by composition with the Euler map (1). Any point of the deformed rod in space $s \in[0, L]$ and time $t \in[0, T]$ is addressed by the map $\mathcal{A}(s) \ni \xi \mapsto x(s, t)+\xi_{1} d^{1}(p(s, t))+\xi_{2} d^{2}(p(s, t))$. The parameter $s \in[0, L]$ is the arc length of the undeformed rod centerline, $L>0$ is the total undeformed arc length and $\mathcal{A}(s) \subset \mathbb{R}^{2}$ for $s \in[0, L]$ is a bounded, connected coordinate domain for the coordinates $\xi=\left(\xi_{1}, \xi_{2}\right) \in \mathcal{A}(s)$ in the cross section, which is assumed rigid and plane throughout the deformation. In the terminology of classical differential geometry, the object $(x(\cdot, t),(\Lambda \circ p)(\cdot, t))$ constitutes a so-called smooth 'framed curve' [12]. For a quaternion $p=p_{0}+\hat{p}=\Re(p)+\Im(p)=$ $\left(p_{0} \mid p_{1}, p_{2}, p_{3}\right)^{\top} \in \mathbb{H}$, the frame $\Lambda(p)$ is given via the Euler map

$$
\Lambda: \mathbb{H} \rightarrow \mathbb{R} S O(3), \quad p \mapsto\left(d^{1}(p)\left|d^{2}(p)\right| d^{3}(p)\right)=\left(2 p_{0}^{2}-\|p\|^{2}\right) \mathcal{I}+2 \hat{p} \otimes \hat{p}+2 p_{0} \mathcal{E}(\hat{p})
$$

with the alternating skew tensor $\mathcal{E}: \mathbb{R}^{3}=\Im(\mathbb{H}) \rightarrow s o(3)$, which identifies skew tensors in $s o(3)$ with their corresponding axial vectors in $\mathbb{R}^{3}$ via $\mathcal{E}(u) w=u \times w$ for $u, w \in \mathbb{R}^{3}$. We write $u \simeq \mathcal{E}(u)$ for $u \in \mathbb{R}^{3}$. The directors $d^{1}(p)$ and $d^{2}(p)$ span the rigid cross section of the rod. The third director $d^{3}(p)$ is always normal to the cross section and is kept close to the tangent $\partial_{s} x$ of the centerline by shearing. Via $\Lambda$, the unit sphere $\mathbb{S}^{3}$ covers $S O(3)$ exactly two times, the correspondence $\Lambda: \mathbb{S}^{3} /\{ \pm 1\} \rightarrow S O(3)$ is one-to-one and onto [16, 20]. Stretched rotation can be expressed via quaternions as $\Lambda(p) w=p w \bar{p}$ (forward) and $\Lambda(p)^{\top} w=\bar{p} w p$ (backward) for $p \in \mathbb{H}$ and $w \in \Im(\mathbb{H})=\mathbb{R}^{3}$, especially $d^{l}(p)=p e^{l} \bar{p}=\Lambda(p) e^{l}$ for each of the space fixed Euclidean base vectors $e^{1}=i, e^{2}=j$ and $e^{3}=k$ of $\Im(\mathbb{H})=\mathbb{R}^{3}$. Recall that the quaternion product is defined by

$$
p q=p_{0} q_{0}-\langle\hat{p}, \hat{q}\rangle+p_{0} \hat{q}+q_{0} \hat{p}+\hat{p} \times \hat{q} \quad \text { for } p, q \in \mathbb{H},
$$

where $\langle\cdot, \cdot\rangle$ is the inner product in $\mathbb{H}$ and $\cdot \times \cdot$ the outer product in $\mathbb{R}^{3}$. It is convenient and common use to identify $\Im(\mathbb{H})=\mathbb{R}^{3}$, this means, ordinary Euclidean vectors are considered as quaternions with vanishing real parts. We use the symbols $p_{0}=\Re(p)$ resp. $\hat{p}=\Im(p)=\left(p_{1}, p_{2}, p_{3}\right)^{\top}$ to denote the real resp. the imaginary (= vector) part and $\bar{p}=p_{0}-\hat{p}$ to denote the conjugate of a quaternion $p \in \mathbb{H}$. Note that $\bar{p}=\|p\|^{2} p^{-1}$, where $p^{-1}$ stands for the multiplicative inverse of $p$. Thus unit quaternions yield pure rotations without stretching. For more details on the Hamilton quaternion division algebra/skew field, we refer to [16, 20].

In each point along its centerline, a Cosserat rod possesses six degrees of freedom, three translatory and three rotatory ones. These equivalently correspond to the bending $(2 \times)$, torsion, shearing $(2 \times)$ and extensional degrees of freedom. An extensible Kirchhoff rod additionally satisfies the shearing constraints $\Gamma^{1}=\left\langle d^{1}(p), \partial_{s} x\right\rangle=0, \Gamma^{2}=\left\langle d^{2}(p), \partial_{s} x\right\rangle=0$. The cross sections must always stay orthogonal to the centerline tangent. We have four physical degrees of freedom, namely bending $(2 \times)$, torsion and extension. An inextensible Kirchhoff rod additionally satisfies $\left\|\partial_{s} x\right\|=1$, this means that the rod remains parametrised by arc length all the time during deformation. Then the centerline tangent $\partial_{s} x$ equals $d^{3}$. This is obviously equivalent to $\Gamma^{1}=\Gamma^{2}=0$ and $\Gamma^{3}=\left\langle d^{3}(p), \partial_{s} x\right\rangle-1=0$. The total number of remaining physical degrees of freedom is equal to three. This is, the inextensible Kirchhoff rod is a pure bending $(2 \times)$ and torsion rod. For all models, we tacitly assume that the centerline remains regular, i. e. $\partial_{s} x \neq 0$, throughout deformation.

We continue with the internal energies. The total internal potential $\mathcal{V}=\mathcal{V}_{\mathrm{SE}}+\mathcal{V}_{\mathrm{BT}}$ is additively decomposed into shearing and extensional energy $\mathcal{V}_{\mathrm{SE}}$ and bending and torsion energy $\mathcal{V}_{\mathrm{BT}}$. Firstly,

$$
\mathcal{V}_{\mathrm{SE}}=\frac{1}{2} \int_{0}^{L} \Gamma^{\top} C_{\mathrm{SE}} \Gamma \mathrm{d} s, \quad C_{\mathrm{SE}}=\left(\begin{array}{c|ccc}
0 & & \\
\hline & & G A_{1} & \\
& & G A_{2} & \\
& & & E A
\end{array}\right), \quad \Gamma=\bar{p}\left(\partial_{s} x\right) p-k .
$$

$\Gamma=\Im(\Gamma)$ is the material strain vector. $\Gamma^{1}$ resp. $\Gamma^{2}$ are the strains that measure shearing in $d^{1}-$ resp. $d^{2}$-direction, $\Gamma^{3}$ is the strain measuring extension in $d^{3}$-direction. In components, we have 
$\Gamma^{1}=\left\langle d^{1}(p), \partial_{s} x\right\rangle, \Gamma^{2}=\left\langle d^{2}(p), \partial_{s} x\right\rangle, \Gamma^{3}=\left\langle d^{3}(p), \partial_{s} x\right\rangle-1$. Note that shearing and extension are not decoupled in the Cosserat model. $E=E(s)>0$ denotes Young's modulus and $G=G(s)>0$ the shear modulus of the material, $A=A(s)=\iint_{\mathcal{A}(s)} \mathrm{d}\left(\xi_{1}, \xi_{2}\right)$ is the area of the rigid cross section, $A_{1}=\kappa_{1} A$ and $A_{2}=\kappa_{2} A$ denote effective cross section areas with some dimensionless Timoshenko shear correction factors $\kappa_{1}(s), \kappa_{2}(s)>0$, see [13]. Secondly,

$$
\mathcal{V}_{\mathrm{BT}}=\frac{1}{2} \int_{0}^{L} K^{\top} C_{\mathrm{BT}} K \mathrm{~d} s, \quad C_{\mathrm{BT}}=\left(\begin{array}{c|ccc}
0 & & \\
\hline & E I_{1} & & \\
& & E I_{2} & \\
& & & G J
\end{array}\right), \quad K=2 \bar{p} \partial_{s} p .
$$

$K=\Im(K) \simeq \mathcal{E}(K)$ is the material curvature vector, sometimes referred to as the 'Darboux' vector $[1,3,12] . K^{1}$ resp. $K^{2}$ are the curvatures corresponding to bending around the $d^{1}$ - resp. $d^{2}$-axis, $K^{3}$ is the curvature corresponding to torsion around the $d^{3}$-axis. $I_{1}=I_{1}(s)=\iint_{\mathcal{A}(s)} \xi_{2}^{2} \mathrm{~d}\left(\xi_{1}, \xi_{2}\right)$ and $I_{2}=I_{2}(s)=\iint_{\mathcal{A}(s)} \xi_{1}^{2} \mathrm{~d}\left(\xi_{1}, \xi_{2}\right)$ are the geometric moments of inertia of the rigid cross section. $J=J(s)=I_{3}(s)=\iint_{\mathcal{A}(s)}\left(\xi_{1}^{2}+\xi_{2}^{2}\right) \mathrm{d}\left(\xi_{1}, \xi_{2}\right)=I_{1}(s)+I_{2}(s)$ denotes its polar geometric moment. If the cross section is symmetric, we have $I=I_{1}=I_{2}$ and $J=I_{3}=2 I$. In (4), it is assumed that precurvature vanishes identically, i. e. the rod is straight in its undeformed configuration. This just simplifies the exposition. For the handling of precurvature, see [26, 27, 38]. We remark that the choice of quadratic potentials $\mathcal{V}_{\mathrm{SE}}$ resp. $\mathcal{V}_{\mathrm{BT}}$ in the strain measures $\Gamma$ resp. $K$ is based on the presumption that locally the stresses and strains remain small $[28,38]$.

For the dissipative potential, we follow $[1,2,3]$ and choose friction forces resp. moments that are proportional to the strain resp. curvature rates. In the terminology of [3], this is a "viscoelastic model of complexity one'. The contributions to the internal dissipative potential $\mathcal{D}=\mathcal{D}_{\mathrm{SE}}+$ $\mathcal{D}_{\mathrm{BT}}$ are

$$
\mathcal{D}_{\mathrm{SE}}=\frac{1}{2} \int_{0}^{L} \dot{\Gamma}^{\top} D_{\mathrm{SE}} \dot{\Gamma} \mathrm{d} s, \quad \mathcal{D}_{\mathrm{BT}}=\frac{1}{2} \int_{0}^{L} \dot{K}^{\top} D_{\mathrm{BT}} \dot{K} \mathrm{~d} s
$$

with the material strain rate $\dot{\Gamma}=\partial_{t} \Gamma$ and the material curvature rate $\dot{K}=\partial_{t} K$. We assume the constitutive matrices $D_{\mathrm{SE}}$ and $D_{\mathrm{BT}}$ as diagonal and positive definite.

The total kinetic energy $\mathcal{T}=\mathcal{T}_{T}+\mathcal{T}_{R}$ decomposes into two parts, the translatory $\mathcal{T}_{T}$ and the rotatory $\mathcal{T}_{R}$ one,

$$
\mathcal{T}_{T}=\frac{1}{2} \int_{0}^{L} \varrho A\|\dot{x}\|^{2} \mathrm{~d} s, \quad \mathcal{T}_{R}=\frac{1}{2} \int_{0}^{L} \varrho \Omega^{\top} I \Omega \mathrm{d} s, \quad I=\left(\begin{array}{l|l|ll}
0 & & & \\
\hline & I_{1} & & \\
& & I_{2} & \\
& & J
\end{array}\right), \quad \Omega=2 \bar{p} \partial_{t} p .
$$

$\Omega=\Im(\Omega)$ is the material angular velocity vector, or the 'vorticity' vector in $[38,39], \varrho=\varrho(s)>0$ is the material density, $I_{1}, I_{2}$ and $J=I_{3}$ are as above, and we identify $\Omega \simeq \mathcal{E}(\Omega)$. A short computation with the $p$ dependent $4 \times 4$ quaternion mass matrix

$$
\mu(p)=4 \mathcal{Q}(p) I \mathcal{Q}(p)^{\top}, \quad \mathcal{Q}(p)=\left(\begin{array}{r||rrr}
p_{0} & -p_{1} & -p_{2} & -p_{3} \\
\hline p_{1} & p_{0} & -p_{3} & p_{2} \\
p_{2} & p_{3} & p_{0} & -p_{1} \\
p_{3} & -p_{2} & p_{1} & p_{0}
\end{array}\right)=(p \| F(p)) \in \mathbb{R} S O(\mathbb{H})
$$

shows that the rotatory kinetic energy can be rewritten as $\mathcal{T}_{R}=\frac{1}{2} \int_{0}^{T} \varrho \dot{p}^{\top} \mu(p) \dot{p} \mathrm{~d} s$. Details are carried out in $[26,34]$. The quaternion mass matrix $\mu(p)$ satisfies the symmetry property $\mu(-p)=\mu(p)$, which is a consequence of the fact that both $p$ and $-p$ describe the same rotation $\Lambda(p)=\Lambda(-p)$. Kernel and image of $\mu(p)$ are given by $\operatorname{ker} \mu(p)=\mathbb{R} p$ and $\operatorname{im} \mu(p)=\{p\}^{\perp}$. Consequently, we have $\operatorname{rk} \mu(p)=3$. Interestingly and useful later, the columns of $\mathcal{Q}(p)$ are the eigenvectors of $\mu(p)$ to the eigenvalues $0,4 I_{1}, 4 I_{2}$ and $4 J . \mu(p)$ is positive semi-definite with its one singular dimension in radial direction. 
For the Cosserat model, the internal conservative elastic forces $F^{\Gamma}$ and moments $M^{K}$ are derived from the potential energy as $F^{\Gamma}=C_{\mathrm{SE}} \Gamma$ and $M^{K}=C_{\mathrm{BT}} K$. Similarly, the internal dissipative damping forces $F^{\dot{\Gamma}}$ and moments $M^{\dot{K}}$ can be derived from the dissipation potential as $F^{\dot{\Gamma}}=D_{\mathrm{SE}} \dot{\Gamma}$ and $M^{\dot{K}}=D_{\mathrm{BT}} \dot{K}$. The total internal forces and moments are then $F=F^{\Gamma}+F^{\dot{\Gamma}}$ resp. $M=M^{K}+M^{\dot{K}}$. For the Kirchhoff model, things are essentially different. Here the internal forces $F$ - in contrast to the moments $M$ - are not determined constitutively, since they are of reactive nature due to the presence of internal constraints $[1,3,30]$. For the inextensible model for example, where all the components of the strain vector $\Gamma$ must vanish, these constraint forces must be of the form $F=\left(\nabla_{x, p} \Gamma\right)^{\top} \lambda$ with appropriate Lagrange multipliers. This is a consequence of d'Alembert's principle.

In [38], averaging the normal Piola-Kirchhoff tractions and corresponding torques over the cross section of the deformed rod, for given exterior material force densities $\hat{F}=\hat{F}(t)$ (per length) and given exterior material moment densities $\hat{M}=\hat{M}(t)$ (per length), it was shown that the rod must satisfy the following system of nonlinear hyperbolic partial differential equations, called the dynamical balance equations for geometrically exact rods,

$$
\left\{\begin{array}{rl}
\varrho A \ddot{x} & =\partial_{s} f+\hat{f} \\
\varrho(i \dot{\omega}+\omega \times i \omega) & =\partial_{s} m+\partial_{s} x \times f+\hat{m}
\end{array} \quad(s, t) \in[0, L] \times[0, T] .\right.
$$

Here the spatial quantities $\omega=\Lambda \Omega, i=\Lambda I \Lambda^{\top}, f=\Lambda F, \hat{f}=\Lambda \hat{F}, m=\Lambda M$ and $\hat{m}=\Lambda \hat{M}$ are obtained from the corresponding material ones by the push forward operation $\Lambda(p) \cdot=p \cdot \bar{p}$ : $\Im(\mathbb{H}) \rightarrow \Im(\mathbb{H})$. An equivalent formulation of $(8)$ in 'quaternion language', derived in an alternative fashion, can be found in $[26,27]$. By the quaternionic parametrisation of rotations, which involves the constraint of unit length in any case, (8) becomes a partial differential-algebraic equation.

The result of the following Lemma is of utmost importance. Of course, it is well known [6] — and most probably due to Euler. However, we give a very short and compact proof for the reader's convenience.

Lemma 2.1 (Differential equations for $K$ and $\Omega$ ) The equations $K=2 \bar{p} \partial_{s} p$ in $\mathbb{S}^{3}$ and $K \simeq$ $\mathcal{E}(K)=\Lambda^{\top} \partial_{s} \Lambda$ in $S O(3)$ for the curvature $K$, and the equations $\Omega=2 \bar{p} \partial_{t} p$ in $\mathbb{S}^{3}$ and $\Omega \simeq \mathcal{E}(\Omega)=$ $\Lambda^{\top} \partial_{t} \Lambda$ in $S O(3)$ for the angular velocity $\Omega$ are equivalent. Here $\Lambda=\Lambda \circ p$.

Proof: We prove the equivalence for $\Omega$. For an arbitrary, but fixed, vector $w \in \Im(\mathbb{H})$, we compute with (2) and the fact that $\bar{w}=-w$ for $\hat{w}=w$,

$$
\begin{aligned}
\mathcal{E}(2 \bar{p} \dot{p}) w & =2 \bar{p} \dot{p} \times w=2 \Im(\bar{p} \dot{p} \times w-\langle\bar{p} \dot{p}, w\rangle)=2 \Im(\bar{p} \dot{p} w)=\bar{p} \dot{p} w-\overline{\bar{p} \dot{p} w}=\bar{p} \dot{p} w+\overline{\bar{p} \dot{p} \bar{w}} \\
& =\bar{p} \dot{p} w+w \dot{\bar{p}} p=\bar{p}(\dot{p} w \bar{p}+p w \overline{\bar{p}}) p=\bar{p} \partial_{t}(p w \bar{p}) p=\bar{p} \partial_{t}(\Lambda w) p=\bar{p}(\dot{\Lambda} w) p=\Lambda^{\top} \dot{\Lambda} w
\end{aligned}
$$

where ${ }^{\cdot}=\partial_{t}$. Now, if $\Lambda^{\top} \dot{\Lambda}=\mathcal{E}(\Omega)$, it follows that $\Omega=2 \bar{p} \dot{p}$, as $w$ can be chosen arbitrarily.

The reader should note that, interestingly, the situation for $K$ and $\Omega$ is always completely symmetric/analogous, if we look at them in two dimensions $(s, t) \in[0, L] \times[0, T]$. The curvature $K$ is so-to-say the 'angular velocity of $p$ along the space dimension $s$ '. By the use of the horizontal method of lines for the discrete model, evolution $K=2 \bar{p} \partial_{s} p$ forms the basis for spatial discretisation, whereas evolution $\Omega=2 \bar{p} \partial_{t} p$ is solved 'continuously' in time. The following Lemma states that the three classical rod models are objective (or frame-indifferent), which means that $\Gamma, K$ and $\Omega$ are invariant w.r.t. superimposed rigid body motions (translations and rotations), which is essential for 'geometrical exactness'. We will see below that our discrete versions as well enjoy this property.

Lemma 2.2 (Objectivity) The material angular velocity $\Omega=2 \bar{p} \partial_{t} p$, the material curvature $K=2 \bar{p} \partial_{s} p$ and the material strain $\Gamma=\bar{p}\left(\partial_{s} x\right) p-k$ are objective. 
Proof: Examining translations is obvious. If we superimpose a constant rotation $\pi \in \mathbb{S}^{3}$, the associated material curvature $K^{*}$, which is obtained by replacing $p(s, t)$ by $\pi p(s, t)$, becomes $K^{*}=2(\overline{\pi p}) \partial_{s}(\pi p)=2 \bar{p} \bar{\pi} \pi \partial_{s} p=2 \bar{p} \partial_{s} p=K$, since $\bar{\pi} \pi=\|\pi\|^{2}=1$. The components of the forward rotated tangents w.r.t. the global basis $\left(e^{1}, e^{2}, e^{3}\right)=(i, j, k)$ are given by $\partial_{s}(\pi x(s, t) \bar{\pi})$. Thus, the associated material strain $\Gamma^{*}$, which is obtained after rotation by $\pi$, satisfies $\Gamma^{*}=$ $\overline{\pi p}\left(\pi \partial_{s} x \bar{\pi}\right)(\pi p)-k=\bar{p} \bar{\pi} \pi \partial_{s} x \bar{\pi} \pi p-k=\Gamma$.

\section{Discrete geometrically exact rods}

In this section, we present our discrete rod versions, based on a staggered grid finite difference discretisation. An obvious advantage of this staggered approach is that for the discrete strain vector $\Gamma_{n-1 / 2}$, no interpolation is needed, yielding a slim three point formula. Shear locking can be avoided thus easily. Further, a staggered grid discretisation approach is much closer to the geometry of the framed curve: From a discrete differential geometric point of view, discrete curvature $K_{n}$ must be situated on the vertices. These rod models are 'discrete framed curves' $[7,10,27]$.

$$
\Gamma_{n-1 / 2}=\frac{1}{\Delta s_{n-1 / 2}} \bar{p}_{n-1 / 2}\left(x_{n}-x_{n-1}\right) p_{n-1 / 2}-k \rightarrow \begin{aligned}
& \text { midpoint quadrature } \\
& \text { for } \mathcal{V}_{S E}
\end{aligned}
$$

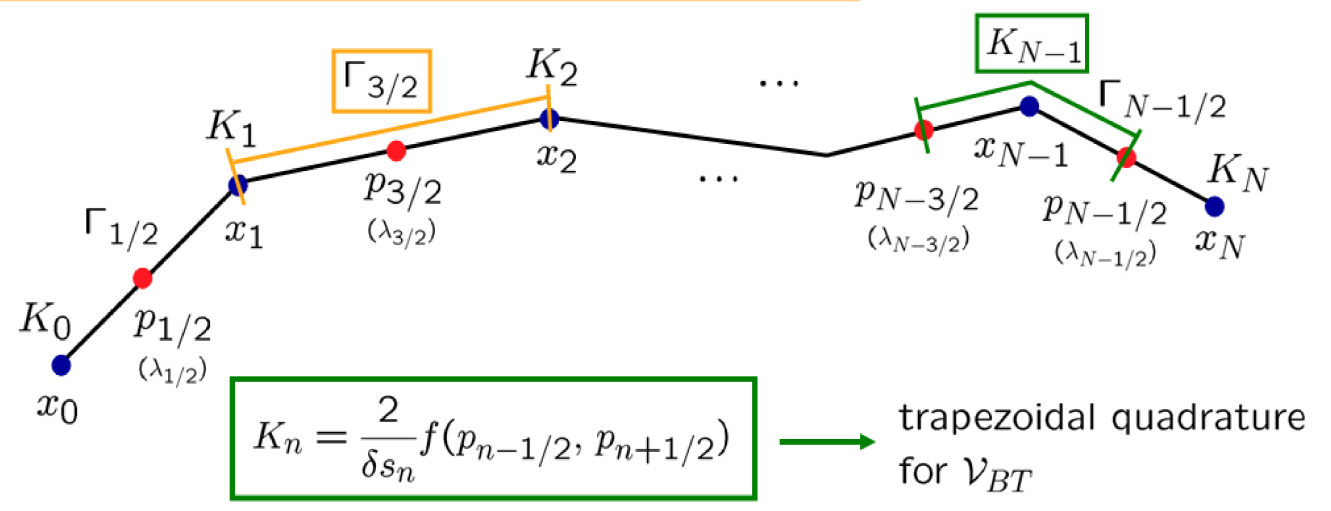

Figure 1: Our discrete differential geometric rod models

Where can this work be situated within the state of the art rod models? The standard approach to handle flexible objects in multibody dynamics simulations [33, 36], which is as well supported by commercial software packages such as SimPACK, ADAMs or ViRTuALLAB, represents flexible structures by vibrational modes, e.g. of Craig and Bampton type [13], that are obtained from numerical modal analysis within the range of linear elasticity. Such methods are suitable and accurate to represent oscillatory response that results from linear response of the flexible structure. Unlike that, our approach is not of modal kind. Each discretised model can be interpreted as a multibody system. So, for example, the inextensible Kirchhoff rod is simply a chain of rigid cylinders connected with bushings that are consistently derived from the continuum equations. But our discretisation approach stands in contrast to the usual way in computational continuum mechanics, where the finite element (FE) approach is favored [9, 11, 14, 21, 23, 38, 39]. The reason for that is, that the main focus in FE is accuracy, not computational efficiency. A very hard problem in geometrically nonlinear FE is the proper interpolation of finite rotations such that objectivity of the strain measures is maintained [14, 31]. (Rigid body motions must not cause additional strains.) This yields extremely technical and sophisticated models with expensive right hand side functions and Jacobians. Our discretisation is motivated from a discrete differential geometric viewpoint. It is much simpler and the objectivity of the smooth models, see Lemma 


\section{2 , is easily maintained.}

We use the horizontal method of lines and start first with the discretisation in space. We subdivide the arc length interval $[0, L]$ into $N$ segments $\left[s_{n-1}, s_{n}\right]$ with the vertices $0=s_{0}<s_{1}<\ldots<$ $s_{N-1}<s_{N}=L$. Together with the midpoints $s_{n-1 / 2}=\left(s_{n-1}+s_{n}\right) / 2$, we have the staggered grid $0=s_{0}<s_{1 / 2}<s_{1}<\ldots<s_{N-1}<s_{N-1 / 2}<s_{N}=L$. The situation is depicted in Figure 1 .

Now we let the discrete translatory degrees of freedom $x_{n}:[0, T] \rightarrow \mathbb{R}^{3}$, i. e. the cross section centroids, live on the vertices, $x_{0}(\cdot) \approx x\left(s_{0}, \cdot\right), \ldots, x_{N}(\cdot) \approx x\left(s_{N}, \cdot\right)$, and the discrete rotatory degrees of freedom $p_{n-1 / 2}:[0, T] \rightarrow \mathbb{H}$, i. e. the quaternions specifying the frame orientations, on the segment midpoints, $p_{1 / 2}(\cdot) \approx p\left(s_{1 / 2}, \cdot\right), \ldots, p_{N-1 / 2}(\cdot) \approx p\left(s_{N-1 / 2}, \cdot\right)$. The corresponding frames $\Lambda\left(p_{n-1 / 2}\right)$ and the directors $d^{l}\left(p_{n-1 / 2}\right)$ live as well on the midpoints. In order to apply clamped boundary rotations properly, virtual ghost quaternions can be introduced, which is a standard technique $[27,29]$. Further, we have to introduce discrete Lagrange multipliers $\lambda_{n-1 / 2}$ : $[0, T] \rightarrow \mathbb{R}^{n_{\lambda}}$, situated as well on the midpoints, $\lambda_{1 / 2}(\cdot) \approx \lambda\left(s_{1 / 2}, \cdot\right), \ldots, \lambda_{N-1 / 2}(\cdot) \approx \lambda\left(s_{N-1 / 2}, \cdot\right)$. The current constraint manifold at time $t \in[0, T]$ is $\left\{q \in \mathbb{R}^{N_{q}}: g(q, t)=0\right\}$, where, for the Cosserat model

$$
g=g(q, t)=\left(\frac{1}{2}\left(\left\|p_{n-\frac{1}{2}}\right\|^{2}-1\right)\right)_{n=1, \ldots, N}, \quad n_{\lambda}=1
$$

for the extensible Kirchhoff model

$$
g=g(q, t)=\left(\begin{array}{c}
\frac{1}{2}\left(\left\|p_{n-\frac{1}{2}}\right\|^{2}-1\right) \\
\Gamma_{n-\frac{1}{2}}^{1}\left(x_{n-1}, p_{n-\frac{1}{2}}, x_{n}, t\right) \\
\Gamma_{n-\frac{1}{2}}^{2}\left(x_{n-1}, p_{n-\frac{1}{2}}, x_{n}, t\right)
\end{array}\right)_{n=1, \ldots, N}, \quad n_{\lambda}=3
$$

for the inextensible Kirchhoff model

$$
g=g(q, t)=\left(\begin{array}{c}
\frac{1}{2}\left(\left\|p_{n-\frac{1}{2}}\right\|^{2}-1\right) \\
\Gamma_{n-\frac{1}{2}}^{1}\left(x_{n-1}, p_{n-\frac{1}{2}}, x_{n}, t\right) \\
\Gamma_{n-\frac{1}{2}}^{2}\left(x_{n-1}, p_{n-\frac{1}{2}}, x_{n}, t\right) \\
\Gamma_{n-\frac{1}{2}}^{3}\left(x_{n-1}, p_{n-\frac{1}{2}}, x_{n}, t\right)
\end{array}\right)_{n=1, \ldots, N}, \quad n_{\lambda}=4
$$

The discrete strains $\Gamma_{n-1 / 2}^{l}=\Gamma_{n-1 / 2}^{l}\left(x_{n-1}, p_{n-1 / 2}, x_{n}\right)$ are explained in (10). In the sequel, we discretise the continuous internal Cosserat energy integrals $\mathcal{V}, \mathcal{T}$ and $\mathcal{D}$ by the use of either midpoint or trapezoidal quadrature, depending on where which quantity is 'at home'. Then, with $q=(x, p) \in \mathbb{R}^{N_{q}}, v=\dot{q} \in \mathbb{R}^{N_{q}}, \lambda \in \mathbb{R}^{N_{\lambda}}$, the discrete potential energy $\mathcal{V}=\mathcal{V}(q, t)$, the discrete kinetic energy $\mathcal{T}=\mathcal{T}(q, v, t)=\frac{1}{2} v^{\top} \mathcal{M}(q) v$, the discrete dissipative potential $\mathcal{D}=\mathcal{D}(q, v, t)$, the Lagrangian function $\mathcal{L}=\mathcal{L}(q, v, t)=\mathcal{T}(q, v, t)-\mathcal{V}(q, t)-g(q, t)^{\top} \lambda$ and prescribed exterior forces $\phi(t) \in \mathbb{R}^{N_{q}}$, the variational principle $\delta \int_{0}^{T} \mathcal{L} \mathrm{d} t+\int_{0}^{T}\left(\phi-\partial_{v} \mathcal{D}\right) \delta q \mathrm{~d} t$ yields a Lagrangian mechanical system $[4,22]$ with holonomic constraints as Euler-Lagrange equations,

$$
\left\{\begin{aligned}
\dot{q} & =v \\
\mathcal{M}(q) \dot{v} & =\psi(q, v, t)-\mathcal{G}(q, t)^{\top} \lambda \\
0 & =g(q, t)
\end{aligned}\right.
$$

Here the right hand side forces are given by $\psi(q, v, t)=\phi(t)-\partial_{q} \mathcal{V}-\partial_{v} \mathcal{D}+\partial_{q} \mathcal{T}-\partial_{q}(\mathcal{M} v) v \in \mathbb{R}^{N_{q}}$, the constraint gradient is $\mathcal{G}(q, t)=\nabla_{q} g(q, t) \in \mathbb{R}^{N_{\lambda} \times N_{q}}$ and the mass matrix $\mathcal{M}(q) \in \mathbb{R}^{N_{q} \times N_{q}}$ is positive semi-definite and symmetric. If no boundary values are prescribed, we have $q, v \in \mathbb{R}^{N_{q}}$, $\lambda \in \mathbb{R}^{N_{\lambda}}$, where $N_{q}=(N+1) n_{x}+N n_{p}, N_{\lambda}=N n_{\lambda} n_{x}=3, n_{p}=4, n_{\lambda}$ as above and $N$ is the number of rod segments.

Before we start with the discrete energies, we give some comments about notation. We consequently use the notation ${ }_{n}$ for quantities on the vertices $s_{n}$; here $n$ always ranges from 0 to $N$. We use the notation ${ }_{n-1 / 2}$ for quantities that are situated on the midpoints $s_{n-1 / 2}$; here $n$ always 
ranges from 1 to $N$. The weight factors for the midpoint rule are the segment lengths, denoted by $\Delta s_{n-1 / 2}=s_{n}-s_{n-1}$. Likewise, the weights for the trapezoidal rule are the lengths of the bucked segments $2 \delta s_{0}=\Delta s_{1 / 2}, 2 \delta s_{n}=\Delta s_{n-1 / 2}+\Delta s_{n+1 / 2}$ and $2 \delta s_{N}=\Delta s_{N-1 / 2}$. See Figure 1 .

We start with the discrete version of the potential energies. We plug the discrete material strains $\Gamma_{n-1 / 2}$ on the segment midpoints, with a simple central difference for the discrete tangents. The potential extensional and shearing energy (3) is approximated with the midpoint rule,

$$
\mathcal{V}_{\mathrm{SE}}=\frac{1}{2} \sum_{n=1}^{N} \Gamma_{n-\frac{1}{2}}^{\top} C_{\mathrm{SE}} \Gamma_{n-\frac{1}{2}} \Delta s_{n-\frac{1}{2}}, \quad \Gamma_{n-\frac{1}{2}}=\bar{p}_{n-\frac{1}{2}} \frac{\Delta x_{n-\frac{1}{2}}}{\Delta s_{n-\frac{1}{2}}} p_{n-\frac{1}{2}}-k,
$$

where $\Delta x_{n-1 / 2}=x_{n}-x_{n-1}$. The discrete strain $\Gamma_{n-1 / 2}$ locally depends on $x_{n-1}, x_{n}$ and $p_{n-1 / 2}$, no interpolation is necessary, shear locking is avoided. It is not hard to show that this special choice for the discrete material strain is objective. The proof is analogous to the continuous case, see Lemma 2.2. We plug the discrete curvatures $K_{n}$ on the vertices and, consequently, approximate the potential bending and torsion energy (4) with the trapezoidal rule

$$
\mathcal{V}_{\mathrm{BT}}=\frac{1}{2} \sum_{n=0}^{N} K_{n}^{\top} C_{\mathrm{BT}} K_{n} \delta s_{n}, \quad K_{n}=K_{n}\left(\delta s_{n}, p_{n-\frac{1}{2}}, p_{n+\frac{1}{2}}\right),
$$

where the discrete material curvatures $K_{n}$ depend on $p_{n-1 / 2}$ and $p_{n+1 / 2}$. The choice of a discrete curvature measure is by no means unique. We propose several choices from literature $[6,7,10,24$, $27,32,40]$, which can be expressed in terms of the material unit axis $u_{n}$ and the material angle $\varphi_{n}$ of difference rotation. Typical examples are

$$
K_{n}=\frac{\varkappa}{\delta s_{n}} \sin \left(\frac{\varphi_{n}}{\varkappa}\right) u_{n}, \quad K_{n}=\frac{\varkappa}{\delta s_{n}} \tan \left(\frac{\varphi_{n}}{\varkappa}\right) u_{n} \quad \text { or } \quad K_{n}=\frac{1}{\delta s_{n}} \varphi_{n} u_{n} .
$$

Here $\varkappa \in \mathbb{N}$ denotes any natural number. The first two curvatures correspond to vectorial parametrisations with the sine resp. tangent generator family in [6]. The third curvature choice can be considered as the limit case for $\varkappa \rightarrow \infty$ [32]. They all satisfy $K_{n}=\mathcal{O}\left(\varphi_{n} / \delta s_{n}\right)$ for $\left|\varphi_{n}\right| \ll 1$. We briefly sketch the simplest choice in (12), namely the one that corresponds to the sine generator family for $\varkappa=2$, already proposed in [40]. That curvature, $K_{n}=2 \sin \left(\varphi_{n} / 2\right) u_{n}$, is obtained with a simple secant interpolation $p_{n}=\left(p_{n-1 / 2}+p_{n+1 / 2}\right) / 2$ and a simple finite central difference $\delta p_{n}=p_{n+1 / 2}-p_{n-1 / 2}$ as $K_{n}=2 \bar{p}_{n} \delta p_{n} / \delta s_{n}$. Objectivity for that discrete curvature can be proven in the same way as in the smooth case, see Lemma 2.2. Unfortunately, this 'naive' approach suffers from poor stability properties [27].

The details for the more sophisticated curvatures in (12) are special topics of elastostatics for rods and go beyond the scope of the present paper. The basic difficulty for a proper discrete curvature definition is to interpolate rotations in a proper, objective way at acceptable numerical costs. For thorough discussions on that topic, we refer to [31]. Approaches that use Cayley transformation instead of interpolation are as well possible [24]. For each of the proposed curvatures in (12), it is possible to show that they are objective/frame-indifferent. The underlying differential geometric reason is that the quaternion unit sphere $\mathbb{S}^{3}$ is completely isotropic — or 'fair' —, in the sense that no special direction is preferred $[16,20]$.

The discretisation of the dissipative potential must be be consistent with the discretisation of the potential energies. We let

$$
\mathcal{D}_{\mathrm{SE}}=\frac{1}{2} \sum_{n=1}^{N} \dot{\Gamma}_{n-\frac{1}{2}}^{\top} D_{\mathrm{SE}} \dot{\Gamma}_{n-\frac{1}{2}} \Delta s_{n-\frac{1}{2}}, \quad \mathcal{D}_{\mathrm{BT}}=\frac{1}{2} \sum_{n=0}^{N} \dot{K}_{n}^{\top} D_{\mathrm{BT}} \dot{K}_{n} \delta s_{n}
$$

with the discrete material strain rates $\dot{\Gamma}_{n-1 / 2}=\dot{\Gamma}_{n-1 / 2}\left(x_{n-1}, x_{n}, p_{n-1 / 2}, \dot{x}_{n-1}, \dot{x}_{n}, \dot{p}_{n-1 / 2}\right)$ and the discrete material curvature rates $\dot{K}_{n}=\dot{K}_{n}\left(p_{n-1 / 2}, p_{n+1 / 2}, \dot{p}_{n-1 / 2}, \dot{p}_{n+1 / 2}\right)$.

As the primary unknowns are ordered in alternating fashion, we discretise the kinetic energy summands in (6) by the trapezoidal rule and the midpoint rule

$$
\mathcal{T}_{T}=\frac{1}{2} \sum_{n=0}^{N} \varrho_{n} A_{n}\left\|\dot{x}_{n}\right\|^{2} \delta s_{n}, \quad \mathcal{T}_{R}=\frac{1}{2} \sum_{n=1}^{N} \varrho_{n-\frac{1}{2}} \dot{p}_{n-\frac{1}{2}}^{\top} \mu\left(p_{n-\frac{1}{2}}\right) \dot{p}_{n-\frac{1}{2}} \Delta s_{n-\frac{1}{2}} .
$$


That way, we lump the translatory masses to the vertices and the rotatory quaternion masses to the midpoints. The discrete material angular velocities $\Omega_{n-1 / 2}=2 \bar{p}_{n-1 / 2} \dot{p}_{n-1 / 2}$ as well belong to the midpoints. The mass matrix $\mathcal{M}(q)$ of the system is block diagonal with alternating $3 \times 3$ (translatory, diagonal and constant) and $4 \times 4$ (rotatory, full and position-dependent) blocks. Each summand in (13) can be interpreted as the rotatory energy of a rigid body with physical moments of inertia equal to $\mathfrak{I}_{1}=\varrho \Delta s I_{1}, \mathfrak{I}_{2}=\varrho \Delta s I_{2}$ and $\mathfrak{I}_{3}=\varrho \Delta s I_{3}$, which are the physical moments of inertia of discs with vanishing thickness $[27,34]$.

It can be seen that in the equidistant case, the discretisation is of second order, which is illustrated by the numerical test results in Figure 2. The asymptotic slopes of the maximum errors in the double logarithmic plot equal two. Positions, velocities and accelerations do converge quadratically, provided that the problem has a smooth solution and that the initial and boundary data are provided consistently. This is not surprising, since - in the equidistant case - both the midpoint and the trapezoidal rule (for the discrete energies) are of second order, and the finite differences (for the discrete strain measures), which are in fact central differences, are of second order, too.

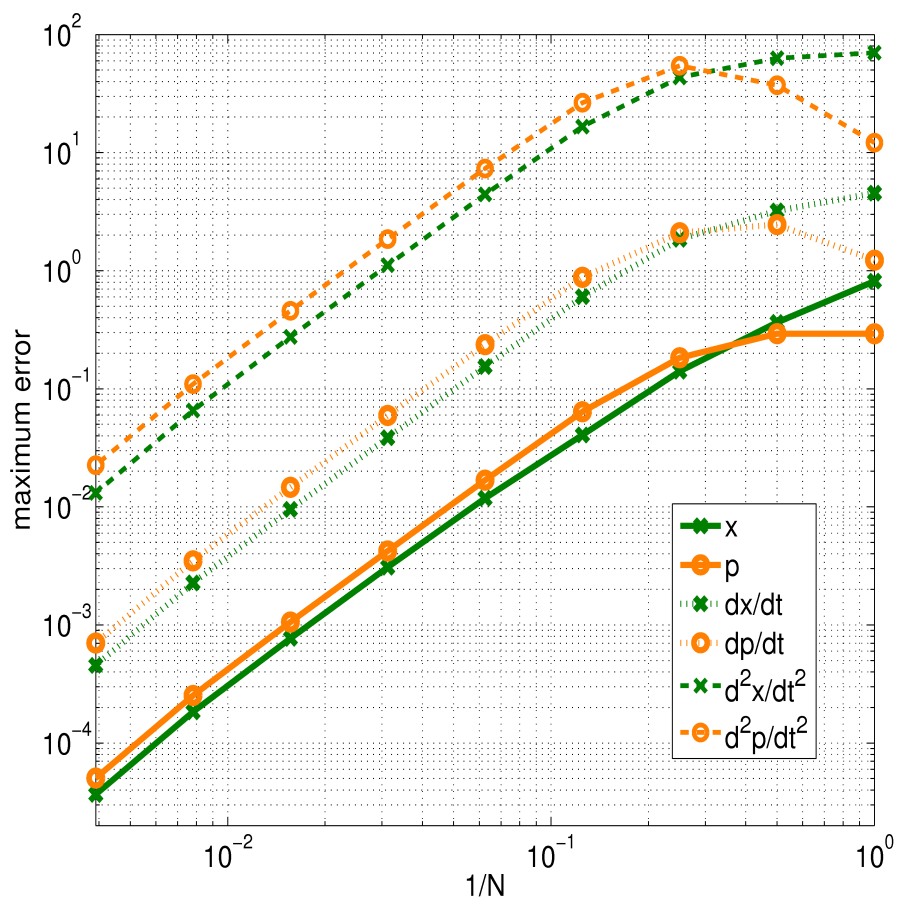

Figure 2: Quadratic convergence for $N \rightarrow \infty$ of the discretisation schemes in the equidistant case. The plot displays the results for a swinging - at one end translatory fixed - damped rubber Cosserat rod in formulation (14) with projection to avoid the drift-off effect. This is Test 1 in [27] and a standard beam/rod benchmark [35].

The reader should observe that, intrinsically in the model, there are many skew symmetries due to the quaternions. The exploitation of these is one reason, why the right hand side of the models can be implemented with extremely few elementary arithmetic operations, see Table 2. Another benefit of quaternions is that they comprise quadratic instead of trigonometric expressions, see (1). So there is no vast blow-up for the Jacobians or Hessians.

Tests versus detailed finite element solutions have been presented in [27], where as well the performance of the model has been demonstrated. It can be shown that the approach is a consistent discretisation to the smooth balance equations (8), see [26, 27]. Yet, formal proofs of stability and convergence are still open questions. 


\begin{tabular}{|c||c|c||c|c||c|}
\hline OPS & sine generator & sine generator & tangent generator & tangent generator & limit case \\
& $\varkappa=2$ & $\varkappa=4$ & $\varkappa=2$ & $\varkappa=4$ & $\varkappa \rightarrow \infty$ \\
\hline \hline+ & $174 N+34$ & $+10 N+10$ & $+11 N+10$ & $+11 N+11$ & $+37 N+37$ \\
- & $111 N+36$ & $+15 N+15$ & $+15 N+15$ & $+27 N+27$ & $+03 N+03$ \\
$*$ & $289 N+90$ & $+39 N+39$ & $+40 N+39$ & $+36 N+36$ & $+72 N+72$ \\
$/$ & $3 N+03$ & $+30 N+30$ & $+30 N+30$ & $+31 N+31$ & $+03 N+03$ \\
2 & $4 N+00$ & $+00 N+00$ & $+00 N+00$ & $+00 N+00$ & $+01 N+01$ \\
\hline$\sqrt{ }$ & $0 N+00$ & $+00 N+00$ & $+00 N+00$ & $+01 N+01$ & $+01 N+01$ \\
arccos & $0 N+00$ & $+00 N+00$ & $+00 N+00$ & $+00 N+00$ & $+01 N+01$ \\
\hline
\end{tabular}

Table 2: Operation counts for the Cosserat right hand side $\Phi$ for different curvature types in absolute coordinates and ODE form $(\dot{q}, \dot{v})=\Phi(q, v, t)$. (The Jacobian $\partial \Phi / \partial(q, v)$ is about twelve times as expensive. For the Kirchhoff models, these counts are similar.)

\section{Numerical problems in time integration}

In this section, we discuss several topics that are important for proper, efficient time integration of the semi-discrete system (9). The exposition in this section relies on the discrete differential geometric model presented before. System (9) is known to be a differential algebraic equation of index 3, see [4, 22]. It is also well known that the numerical solution of the index-3 system involves difficulties such as poor convergence of Newton's method [4, 17, 22]. Thus, we reduce the index to 1 , yielding

$$
\left(\begin{array}{ccc}
\mathcal{I} & 0 & 0 \\
0 & \mathcal{M}(q) & \mathcal{G}(q, t)^{\top} \\
0 & \mathcal{G}(q, t) & 0
\end{array}\right)\left(\begin{array}{c}
\dot{q} \\
\dot{v} \\
\lambda
\end{array}\right)=\left(\begin{array}{c}
v \\
\psi(q, v, t) \\
-g^{\mathrm{II}}(q, v, t)
\end{array}\right)
$$

Here all the right hand side terms that emerge from time differentiation are collected in the function $g^{\mathrm{II}}(q, v, t)=\partial_{q q}^{2} g(q, t)[v, v]+2 \partial_{t} G(q, t) v+\partial_{t t}^{2} g(q, t)$. Solving (14) for $(\dot{q}, \dot{v}, \lambda)$ and discarding the equation for the Lagrange multipliers, we receive an $\operatorname{ODE} \dot{u}=\Phi(u, t)$ for the unknowns $u=(q, v)$.

The following Null Space Coordinate Formulation is an interesting alternative, which is especially simple and will turn out — at least for Cosserat rods — to be superior to (14). The adaptation of this technique to the Kirchhoff models seems promising, but is an open topic. We summarise the simple and elegant idea of null space coordinates [8, 17]. It is some kind of compromise between absolute and relative (minimal) coordinate formulations. On position level, we keep the absolute coordinates $q \in \mathbb{R}^{N_{q}}$ (not minimal), but on velocity level, instead on $v \in \mathbb{R}^{N_{q}}$, a minimal number of unknowns $\nu \in \mathbb{R}^{N_{\mathrm{DOF}}}$, where $N_{\mathrm{DOF}}=N_{q}-N_{\lambda} \geq 0$, is chosen. These minimal unknowns $\nu=\left(\nu_{1}, \ldots, \nu_{N_{\mathrm{DOF}}}\right)$ are the coefficients of the tangential part of the velocity $v$ in the expansion w.r.t an appropriate basis of the current tangential space at $q$. Note that in contrast to the current manifold $\left\{q \in \mathbb{R}^{N_{q}}: g(q, t)=0\right\}$, the current tangential space $\operatorname{ker} \mathcal{G}(q, t)=\left\{\xi \in \mathbb{R}^{N_{q}}: G(q, t) \xi=0\right\}$ is a linear space.

Starting with the Lagrangian system (9), we expand the tangential component of $v$ with respect to a basis $\phi^{1}(q, t), \ldots, \phi^{N_{\mathrm{DOF}}}(q, t) \in \mathbb{R}^{N_{q}}$ of the tangential space of the current constraint manifold,

$$
v=\dot{q}=\sum_{l=1}^{N_{\mathrm{DOF}}} \nu_{l} \phi^{l}(q, t)-\mathcal{G}(q, t)^{\top}\left(\mathcal{G}(q, t) \mathcal{G}(q, t)^{\top}\right)^{-1} \partial_{t} g(q, t)=: \mathcal{F}(q, t) \nu-\varphi(q, t)
$$

We assume the Grübler condition that $\operatorname{rk} \mathcal{G}^{\top}=N_{\lambda}$ is maximal, excluding singular (especially redundant) constraints. Since $\operatorname{ker} \mathcal{G}(q, t)=\operatorname{im} \mathcal{F}(q, t)$, the constraint on the level of velocity is satisfied exactly,

$$
\dot{g}=\mathcal{G} \dot{q}+\partial_{t} g=\mathcal{G}\left(\mathcal{F} \nu-\mathcal{G}^{\top}\left(\mathcal{G} \mathcal{G}^{\top}\right)^{-1} \partial_{t} g\right)+\partial_{t} g \equiv 0 .
$$

Now, the constraint forces $\mathcal{G}(q, t)^{\top} \lambda$ are eliminated, if we multiply the dynamical equations of (9) 
with $\mathcal{F}(q, t)^{\top}$ from the left. We receive the null space coordinate formulation

$$
\left\{\begin{aligned}
\dot{q} & =\mathcal{F}(q, t) \nu-\varphi(q, t) \\
\mathcal{F}(q, t)^{\top} \mathcal{M}(q) \mathcal{F}(q, t) \dot{\nu} & =\mathcal{F}(q, t)^{\top}(\psi(q, \dot{q}, t)+\mathcal{M}(q)(\dot{\varphi}(q, \dot{q}, t)-\dot{\mathcal{F}}(q, \dot{q}, t) \nu)) \\
0 & =g(q, t)
\end{aligned}\right.
$$

The first line consists of $N_{q}$ equations (as for absolute coordinates), the second line of $N_{\text {Dof }}$ equations (as for relative coordinates). The latter is minimal, because it contains exactly as many equations as are present in the physical model, namely $N_{\text {DOF }}=\operatorname{dim}\left\{q \in \mathbb{R}^{N_{q}}: g(q, t)=0\right\}=$ $\operatorname{dim} \operatorname{ker} \mathcal{G}(q, t)=\operatorname{rk} \mathcal{F}(q, t)$. System (17) is overdetermined, as we have $N_{q}$ unknowns $q$ and $N_{\text {DoF }}$ unknowns $\nu$. System $\left(17_{1,2}\right)$ yields an $\operatorname{ODE}(\dot{q}, \dot{\nu})=\Psi(q, \nu, t)$ of dimension $N_{q}+N_{\text {Dof }}$ with the solution invariant $\left(17_{3}\right)$, see [18]. The null space coordinate method is equivalent to solving the corresponding index-2 version of (9) in ODE form, see [17].

We now start discussing numerical problems in time integration, which are summarised in the lower part of Table 1 .

(a) Kinematic singularities. Unfortunately, the inextensible Kirchhoff model, which is a pure bending and torsion rod, contains kinematic singularities. A singularity is obviously given, if such a rod is straight, not predeformed, but clamped at the boundaries: Traction at both ends is forbidden, pressure on both sides results in instantaneous buckling. (Not to mention that the critical Eulerian buckling loads can be predicted precisely.) The existence of such singularities does not depend on the special discretisation or coordinate formulation, it is an intrinsic property already of the smooth model. (In terms of (9), these singularities manifest themselves in the fact that $\mathcal{G}(q, t)^{\top}$ is not of full rank $N_{\lambda}$, i. e. the Grübler condition is not satisfied.) So unfortunately, for industrial applications as the modeling of deformable cables, where only bending and torsion phenomena are of interest, the use of the inextensible Kirchhoff model, which - as we will see does not behave numerically stiff and allows the largest time stepsizes, makes additional tedious handling of such singular configurations necessary.

(b) Handling of the mass- and constraint matrices. In the Cosserat model, translatory and rotatory parts in (14) decouple. For each centroid $x_{n}$ with mass $\varrho_{n} A_{n} \delta s_{n}$, the translatory mass-matrix block is given by a $3 \times 3$ diagonal, constant, state independent block $\varrho_{n} A_{n} \delta s_{n} \mathcal{I}$. For the rotatory part, we fix a segment $\Delta s=\Delta s_{n-1 / 2}, \varrho=\varrho_{n-1 / 2}$ and its quaternion $p=$ $p_{n-1 / 2}$. The constraints on position, velocity and acceleration level are written $2 g=\|p\|^{2}-1=0$, $\dot{g}=G(p) \dot{p}=\langle p, \dot{p}\rangle=0$ and $\ddot{g}=G(p) \ddot{p}+\partial_{p p}^{2} g(p)[\dot{p}, \dot{p}]=\langle p, \ddot{p}\rangle+\|\dot{p}\|^{2}=0$ respectively, where $G(p)=\nabla_{p} g(p)=p^{\top}$. Thus, each rotatory quaternionic mass-constraint block is $5 \times 5$ and has the form

$$
\left(\begin{array}{c|c}
M(p) & G(p)^{\top} \\
\hline G(p) & 0
\end{array}\right)=\left(\begin{array}{c|c}
M(p) & p \\
\hline p^{\top} & 0
\end{array}\right), \quad M(p)=\varrho \Delta s \mu(p)
$$

with the singular quaternion mass $\mu(p)$ from (7). The inverse of (18) exists, iff $p \neq 0$, and has exactly the same form as (18), where $M(p)$ is replaced by $M(p)^{\sharp}=\frac{1}{\varrho \Delta s} \mu^{\sharp}(p)$ with the tangential inverse quaternion mass $\mu^{\sharp}(p)=\frac{1}{4} \mathcal{Q}(p) I^{\sharp} \mathcal{Q}(p)^{\top}, I^{\sharp}=\operatorname{diag}\left(0 \mid 1 / I_{1}, 1 / I_{2}, 1 / J\right)$, which satisfies the property $\mu(p) \mu^{\sharp}(p)=\mu^{\sharp}(p) \mu(p)=\mathcal{I}-p \otimes p$ for $p \in \mathbb{S}^{3}$, which justifies the nomenclature 'tangential inverse', as $\mu^{\sharp}(p) \mu(p) \pi=\mu(p) \mu(p)^{\sharp} \pi=\pi-(p \otimes p) \pi=\pi-\langle p, \pi\rangle p$ for $\pi \in \mathbb{H}$, see [26]. Thus, the structures of (18) and its inverse are completely identical, inversion can be performed at exactly the same numerical complexity [27, 34]. Especially, the inverse of the mass-constraint matrix in (14) is as well block diagonal. Note that for ill-conditioned problems - for example, if the rod is very thin —, the constraints must be scaled appropriately [5, 27].

The situation gets even better, if we apply the aforementioned null space technique to the quaternionic rotatory part. For the special case of Cosserat rods, a decoupled quaternionic rotatory 
block part in index-3 resp. index-1 absolute formulation is given by

$$
\left\{\begin{array} { r l } 
{ \dot { p } } & { = v } \\
{ M ( p ) \dot { v } } & { = \psi ^ { p } - \lambda p } \\
{ 0 } & { = \frac { 1 } { 2 } ( \| p \| ^ { 2 } - 1 ) }
\end{array} \quad , \quad \text { resp. } \quad \left\{\begin{array}{rl}
\dot{p} & =v \\
M(p) \dot{v} & =\psi^{p}-\lambda p \\
\langle p, \dot{v}\rangle & =-\|v\|^{2}
\end{array}\right.\right.
$$

(The reader might equivalently think of the Euler equations for a rigid body.) Here $N_{q}=n_{p}=4$, $N_{\lambda}=n_{\lambda}=1 . \psi^{p} \in \mathbb{H}$ denotes the quaternionic moments acting on $p$, of which only the tangential component is physically relevant, as multiplication with the radially singular $M(p)^{\sharp}$ shows, giving $\dot{v}=M(p)^{\sharp} \psi^{p}-\|v\|^{2} p$. The tangential space $\operatorname{ker} G(p)=\{p\}^{\perp}=\{\pi \in \mathbb{H}:\langle p, \pi\rangle=0\}$ of $\mathbb{S}^{3}$ at $p$ has dimension $N_{\text {DOF }}=3$. An adequate expansion for $v=\dot{p}$ in (15) is

$$
v=\sum_{l=1}^{3} \nu_{l} \phi^{l}(p)=F(p) \nu, \quad \phi^{1}(p)=\left(\begin{array}{c}
-p_{1} \\
p_{0} \\
p_{3} \\
-p_{2}
\end{array}\right), \quad \phi^{2}(p)=\left(\begin{array}{r}
-p_{2} \\
-p_{3} \\
p_{0} \\
p_{1}
\end{array}\right), \quad \phi^{3}(p)=\left(\begin{array}{r}
-p_{3} \\
p_{2} \\
-p_{1} \\
p_{0}
\end{array}\right) .
$$

with the $4 \times 3$ null space matrix $\mathcal{F}(p, t)=F(p)$ from $(7)$. Then we receive the following quaternionic null space formulation for Cosserat rods (or rigid bodies).

Lemma 4.1 (Quaternionic Null Space) The null space formalism, applied to the index-3 problem $\left(19_{1}\right)$ with expansion (20), yields the following special form of (17),

$$
\left\{\begin{array}{rl}
\dot{p} & =p \nu \\
4 \mathfrak{I} \dot{\nu} & =\Im\left(\bar{p} \psi^{p}\right) \\
0 & =\frac{1}{2}\left(\|p\|^{2}-1\right)
\end{array} \quad \mathfrak{I}=\left(\begin{array}{lll}
\mathfrak{I}_{1} & & \\
& \mathfrak{I}_{2} & \\
& & \mathfrak{I}_{3}
\end{array}\right) .\right.
$$

Proof: Firstly, the tangential vectors $\phi^{l}(p)$ are eigenvectors of $M(p)$ with the corresponding eigenvalues $4 \mathfrak{I}_{l}=4 \varrho \Delta s I_{l}$ for $l=1,2,3$. Since $\operatorname{ker} M(p)=\mathbb{R} p$ and $\mathcal{Q}(p) \in\|p\| S O(\mathbb{H})-$ which yields that the system $\left(p, \phi^{1}(p), \phi^{2}(p), \phi^{3}(p)\right)$ is an orthonormal one on $\mathbb{S}^{3}$ - we receive $F(p)^{\top} M(p) F(p)=4 \mathfrak{I}$. Secondly, the following general identities from quaternionic calculus

$$
F(p) w=p w, \quad F(p)^{\top} \pi=\Im(\bar{p} \pi) \quad \text { for } w \in \Im(\mathbb{H}), \pi \in \mathbb{H}
$$

immediately yield $F(p) \nu=p \nu$ and $F(p)^{\top} \psi^{p}=\Im\left(\bar{p} \psi^{p}\right)$. Thirdly, since the system is scleronomic, i. e. $\partial_{t} g \equiv 0$, it follows that $\varphi=\dot{\varphi} \equiv 0$. Finally, from the relations $\left(\partial_{p} \phi^{1}\right) \phi^{2}(p)=$ $-\phi^{3}(p),\left(\partial_{p} \phi^{2}\right) \phi^{3}(p)=-\phi^{1}(p),\left(\partial_{p} \phi^{3}\right) \phi^{1}(p)=-\phi^{2}(p),\left(\partial_{p} \phi^{l}\right) \phi^{l}(p)=-p$ for $l=1,2,3$ and $\dot{F}=\partial_{p}\left(\phi^{1}(p)\left|\phi^{2}(p)\right| \phi^{3}(p)\right) F(p) \nu$, it follows that the term $F^{\top} M \dot{F} \nu \equiv 0$ vanishes.

In fact, with $\left(22_{2}\right)$ and Lemma 2.1 it is easy to see that $\Omega=2 \nu$, so that the null space technique, applied to the quaternionic rotatory formulation, yields a well known mixed formulation for rigid bodies in [17, 34], which uses $(p, \Omega)$ as the primary unknowns. (The reader should note that the term $\Omega \times \mathfrak{I} \Omega$ in the Euler equations, which corresponds to the mass skew derivative $\frac{1}{2} \partial_{p}\left(v^{\top} M v\right)-$ $\partial_{p}(M v) v$, is included in $\psi^{p}$, see [26].) We think, it is an interesting insight that this classical mixed formulation can be embedded into the systematic and general null space coordinate framework. The null space formulation (21) for the Cosserat rod (or a rigid body) has several decisive advantages compared to the full absolute coordinate formulation (19).

(i) The reduced $3 \times 3$ mass matrix $F(p)^{\top} M(p) F(p)=4 \mathfrak{I}$ is diagonal and state-independent, in contrast to $M(p)$ itself, which is $4 \times 4$, full and depends on the quaternion position $p$.

(ii) The constraint is satisfied exactly on velocity level, $\dot{g}=G(p) F(p) \nu \equiv 0$.

(iii) The radial constraint forces $G(p)^{\top} \lambda=\lambda p$, which do not have any physical meaning and which are of no interest, are eliminated exactly. 




Figure 3: Accuracy and Numerical Task. (Absolute formulation $(\dot{q}, \dot{v})=\Phi(q, v, t)$ vs. null space formulation $(\dot{q}, \dot{\nu})=\Psi(q, \nu, t)$ for the Cosserat rod with the solver RADAU5 and $T=10 s$.

(iv) The number of unknowns is reduced from eight for $(p, v)$ to seven for $(p, \nu)$. This is minimal on velocity level, but still singularity free — compared to any three-dimensional — or 'vectorial' [6] — parametrisation of $S O(3)$, which necessarily must have singularities.

(v) The problem of ill-conditioning vanishes, since the Skeel condition of $\mathfrak{I}$ equals one.

(vi) The total number of operations in Table 2 for $\Phi$ is further reduced. This yields a cheaper net right hand side function $\Psi$ (and Jacobian) with $\operatorname{OPS}(\Psi) \leq \frac{13}{14} \mathrm{OPS}(\Phi)$.

Advantage (ii) as well leads to a significantly improved energetic behaviour, as we shall see below. Benefit (iv) leads to an improvement of stepsize selection for all the solvers we have tested. These are the Ode15s, Ode45, Ode23 from the Matlab OdE suite [37], DoPri5, DoP853, Rodas, Seulex, Radau5 and Dassl [22]. In fact, it leads to improved accuracy, see Figure 3 for the implicit method RADAU5 and the same test setup as in Figure 2. The Jacobians for implicit time integration become slightly 'lopsided', as originally $4 \times 4$ blocks become $3 \times 3,3 \times 4$ or $4 \times 3$, see Figure $4(\mathrm{~d})$. Block banded Gaussian elimination can be appropriately adapted [19].

For the Kirchhoff model, the constraints have a substantially more complex structure. Here the constraint gradient $\mathcal{G}(q, t)$ depends on all positions $q$ and the time $t$ (if boundary values are prescribed). So does the mass-constraint matrix on the left-hand side in (14). But the latter is still sparse and banded with upper and lower bandwidths $m=9$ (in the extensible case) resp. $m=10$ (in the inextensible case) for the discrete versions, see Figure 4 (a). Figure 4 (b) nicely demonstrates how the sparsity pattern is destroyed by inversion.

Now for explicit solvers, which can only handle systems of the form

$$
\mathcal{I} \dot{u}=f(t, u) \quad \text { with identity 'mass' } \mathcal{I} \quad(\text { e.g. DoPri5), }
$$

system (14) must be solved for $(\dot{q}, \dot{v}, \lambda)$, so that one can choose $u=(q, v)$ in (23). Banded Gaussian $L U$ factorisation [19] is appropriate with complexity $\mathcal{O}\left(m^{2} N\right)$. In order to avoid this decomposition step, one is restricted to solvers, that may handle linearly implicit systems

$$
\mathcal{A} \dot{u}=f(t, u) \quad \text { with constant 'mass' } \mathcal{A} \quad(\text { e.g. RADAU5) }
$$

or

$$
\mathcal{A}(t, u) \dot{u}=f(t, u) \quad \text { with state dependent 'mass' } \mathcal{A}(t, u) \quad \text { (e.g. DAssL). }
$$




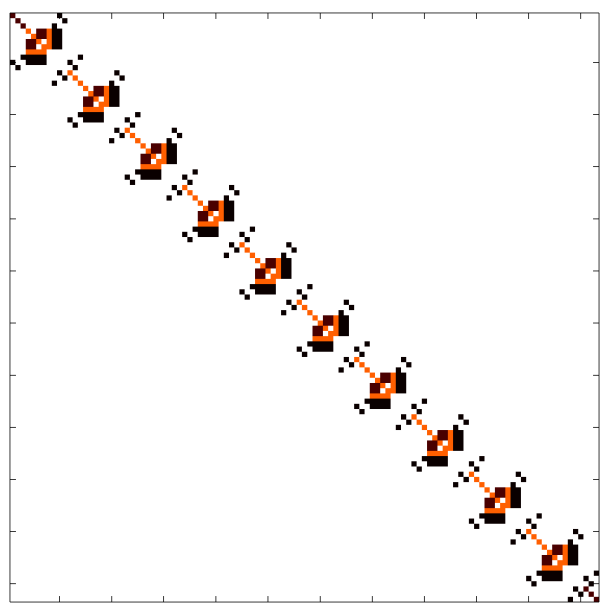

(a)

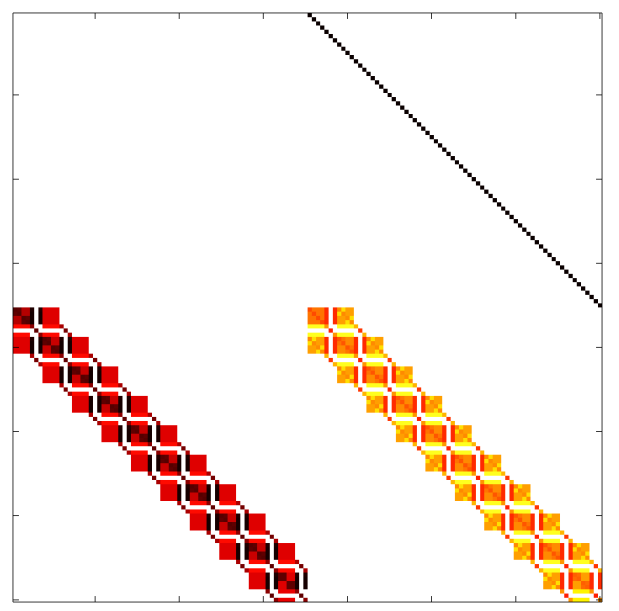

(c)

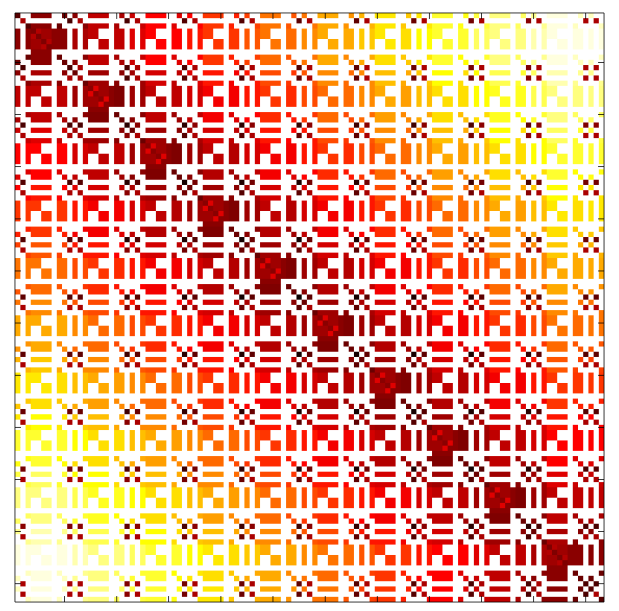

(b)

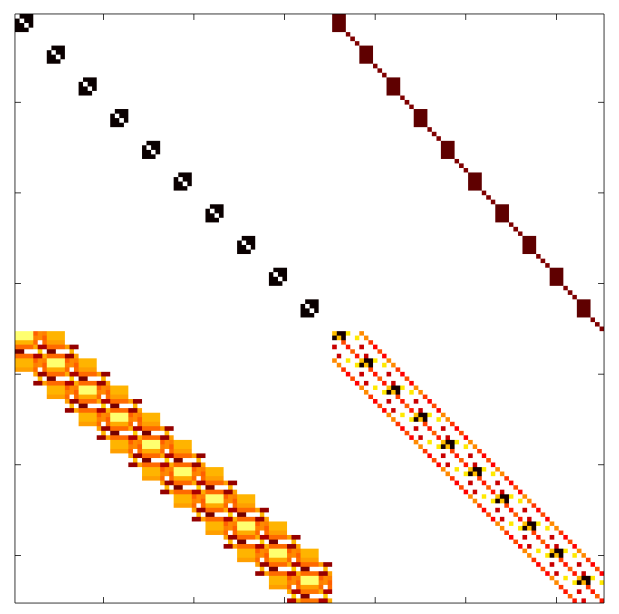

(d)

Figure 4: (a) The mass-constraint matrix for an inextensible Kirchhoff rod. (b) Its Inverse. (c) The Jacobian $\partial(\dot{q}, \dot{v}) / \partial(q, v)$ for a Cosserat rod in absolute coordinates $(\dot{q}, \dot{v})=\Phi(q, v, t)$. (d) The Jacobian $\partial(\dot{q}, \dot{\nu}) / \partial(q, \nu)$ for a Cosserat rod in null space coordinates $(\dot{q}, \dot{\nu})=\Psi(q, \nu, t)$.

Solvers for (25) do not eliminate the Lagrange multipliers $\lambda$ in (14). They solve the full index1 problem with $u=(q, v, \lambda)$. For solvers that may handle linearly implicit systems (24), the accelerations $w=\dot{v}$ must be introduced as additional primary unknowns. Again, one has to solve the index-1 problem with $u=(q, v, w, \lambda)$ with appropriate rescaling in the error estimator [22]. The drawback for use of numerical solution techniques of type (24) or (25) is that these methods are implicit, which in turn, makes the effective evaluation of the Jacobian $\partial f / \partial u$ necessary.

Summarising, in contrast to the Kirchhoff models, the Cosserat model may be combined with any standard solver, the inverse of the mass-constraint (resp. mass) matrix in absolute (resp. null space) coordinates may be evaluated efficiently, as it is sparse and cheap.

(c) Constraint stabilisation. Let us continue with the question, how to avoid the drift-off effect. It is well known that in (14), where the constraint is imposed on acceleration level, the position $q$ (resp. the velocity $v$ ) drifts quadratically (resp. linearly) from the constraint manifold $[4,17,22]$. For the two Kirchhoff models, subsequent (orthogonal) projection of the position $q^{\star}$ and (tangential) projection of the velocity $v^{\star}$ with respect to the pseudo (or 'semi') metric 


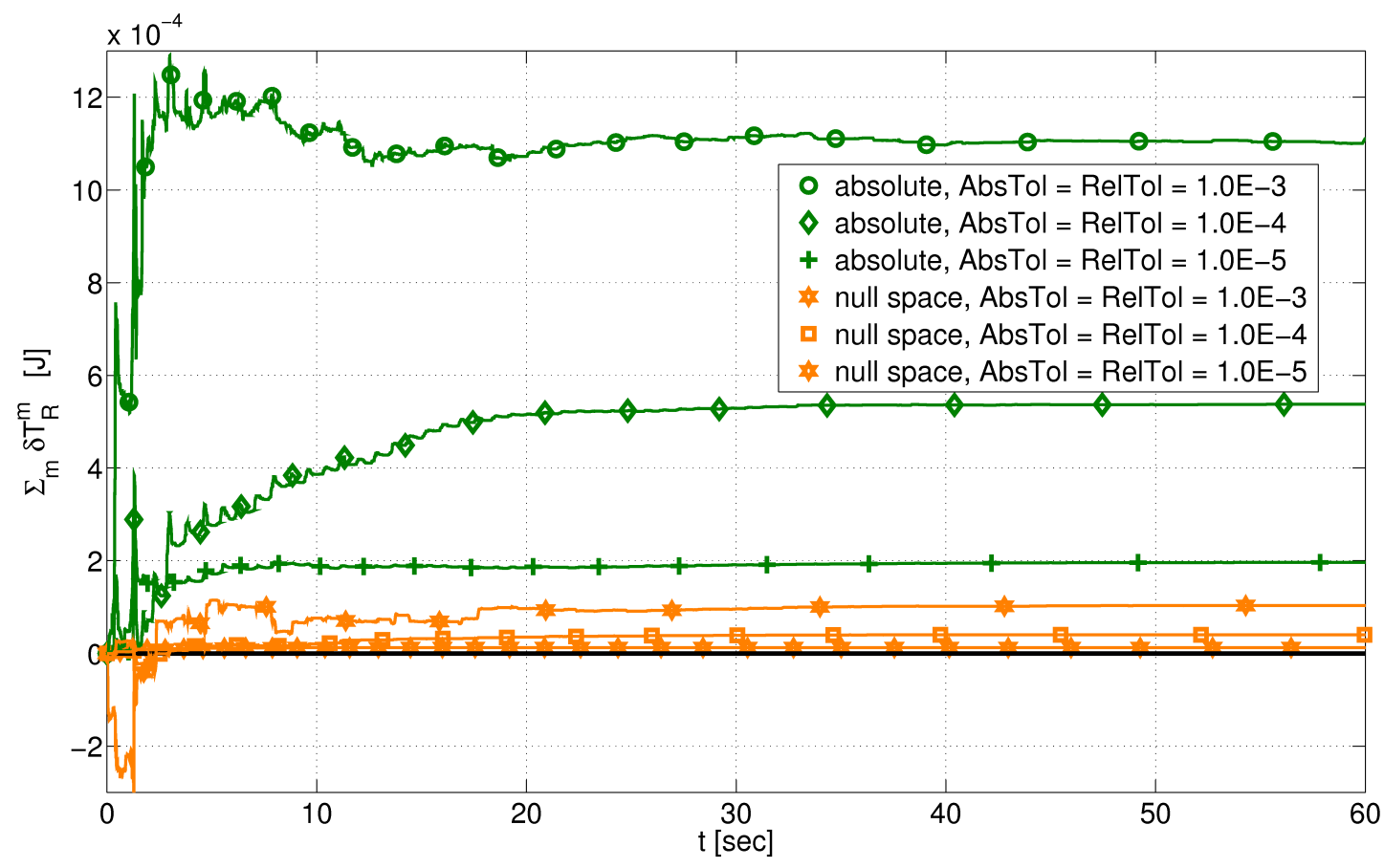

Figure 5: Accumulated rotatory energy defect caused by projection. (Absolute vs. null space coordinate formulation for a swinging Cosserat rubber rod, computed with RADAU5.)

$\langle\mathcal{M}(q) \cdot, \cdot\rangle=\cdot{ }^{\top} \mathcal{M}(q) \cdot$ can be applied $[22]$. This leads to the systems

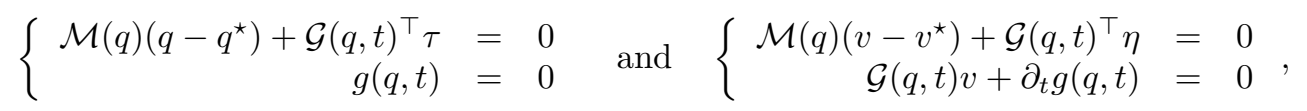

which must be solved consecutively for $(q, \tau)$ and $(v, \eta)$, where $\eta, \tau \in \mathbb{R}^{N_{\lambda}}$ denote additional Lagrange multipliers [22]. The first system $\left(26_{1}\right)$ is nonlinear and must therefore be solved iteratively. Typically, two or three iterations with a simplified Newton method are sufficient, if the projection is applied after each accepted time integration step. The Jacobian for the simplified Newton method is kept constant during iteration. It equals the mass-constraint matrix with the structure displayed in Figure 4 (a). The second system $\left(26_{2}\right)$ is linear and can be solved in one step. Note that in the same way, at the very beginning of the dynamical simulation for $t=0$, consistent initial values can be obtained from inconsistent ones.

For the Cosserat model, things are much easier, since translatory and rotatory parts decouple. Here, for the quaternionic rotatory part, subsequent projection

$$
p=\frac{1}{\left\|p^{\star}\right\|} p^{\star} \quad \text { (of position) } \quad \text { and } \quad v=v^{\star}-\left\langle p, v^{\star}\right\rangle p \quad \text { (of velocity) }
$$

is especially cheap, and no iterative procedure is required. Since ker $M(p)=\mathbb{R} p$, one sees that $(27)$ is embedded into the general framework (26) with $\eta=\tau=0$. Easy and efficient implementations of the projection method, however, are restricted to one step integration methods. For higher order BDF/NDF methods, non-trivial modifications in the core solver, e.g. DASSL [22] or ODE15s [37], are necessary [4].

Another stabilisation technique - already on the model level — is the Baumgarte method [22], where the linear combination $\ddot{g}+2 r \dot{g}+\omega^{2} g=0$ with parameters $\omega, r>0$ is imposed as constraint instead of $g=0, \dot{g}=0$ or $\ddot{g}=0$. It is well known that the Baumgarte method may introduce additional artificial stiffness into the system [22]. The Cosserat model already is (and behaves 




Figure 6: DoPRI5 stepsizes for undamped rubber rods. $\left(\mathrm{ABSTOL}=\mathrm{RELTOL}=1.0 \mathrm{E}^{-3}\right)$

numerically) very stiff, as we will see. So here, Baumgarte stabilisation works surprisingly good, since $\omega$ and $r$ can be chosen sufficiently large without effectively increasing the stiffness of the model [27]. For the Kirchhoff models, which are not as stiff, the Baumgarte method cannot be recommended, as the solver performance is strongly decreased, if the Baumgarte parameters are tightened, i. e. for $\omega, r \gg 1$.

It is known that stabilisation of velocity is more crucial than stabilisation of position [22]. The projection and the Baumgarte stabilisation methods have in common that they may dissipate energy. Considering the projection technique, it is usually the projection of velocity that is the dominant energy consuming process. We point out that for the Cosserat model and both formulations $\left(19_{2}\right)$ and $(21)$ the projection $\left(27_{2}\right)$ does not consume kinetic energy. In formulation $\left(19_{2}\right)$, we have $\left(v^{\star}\right)^{\top} M(p) v^{\star}=v^{\top} M(p) v$, because of $\operatorname{ker} M(p)=\mathbb{R} p$. In formulation $\left(27_{2}\right)$, the velocity $v=T(p) \nu$ - by construction - always $i s$ - up to round-off errors — perfectly tangential to $\mathbb{S}^{3}$ and there is no need to project it. However, the projection of position $\left(27_{1}\right)$ in fact does affect the rotatory kinetic energy. Let $T_{R}^{\star}=\frac{1}{2}\left(v^{\star}\right)^{\top} M\left(p^{\star}\right) v^{\star}, T_{R}=\frac{1}{2} v^{\top} M(p) v$ and $\delta T_{R}=T-T^{\star}$ the defect caused by projection. (We have just seen that $2 T_{R}=\left(v^{\star}\right)^{\top} M(p) v^{\star}$.) Now if the quaternion has drifted to the outside of $\mathbb{S}^{3}$, i. e. $\left\|p^{\star}\right\|>\|p\|=1$, then $\delta T_{R}<0$. If it has drifted to the inside of $\mathbb{S}^{3}$, i. e. $\left\|p^{\star}\right\|<\|p\|=1$, then $\delta T_{R}>0$. Note that the mass is radially sensitive, so that $\left\|p^{\star}\right\|^{2} M(p)=M\left(p^{\star}\right)$. Now in null space formulation (21), the drift-off in $p$ is only linear (not quadratic) in the long run. And it is usually much smaller in each time step than in formulation $\left(19_{2}\right)$. That's why the null space coordinate formulation enjoys a better energetic behaviour. Figure 5 displays the numerical defect accumulation $\sum_{m} \delta T_{R}^{m}$ along the simulated time interval $[0, T]$ for the same setup as in Figures 2 and 3, computed with RADAU5. It clearly demonstrates that formulation $(21)$ is superior to formulation $\left(19_{2}\right)$. In null space coordinates, even for coarse integrator tolerances, sufficiently good energy conservation is obtained in practice.

All this, of course, applies as well to quaternionic rigid bodies. These observations give additional good reasons - compared to those in [34], which are just concerning numerical right hand side costs - , why the null space technique definitively ought to be preferred. 
(d) Numerical stiffness. The last topic in this paper is devoted to the crucial question of numerical stiffness. Let us consider a smooth Cosserat rod. For simplicity, we assume a homogeneous rod with symmetric cross section, i. e. $I:=I_{1}=I_{2}, \kappa:=\kappa_{1}=\kappa_{2}$. In order to estimate the stiffnesses for the DOF 'shearing', 'extension', 'bending' and 'torsion' in dynamics, this observation is not sufficient. So we have to look at the frequencies contained in the model in the undamped case. It is shown in [13] for linear Timoshenko beams - the linearised version of the Cosserat rod - that the eigenfrequencies for 'extension', 'bending' and 'torsion' are of orders $\mathcal{O}\left(L^{-1} \sqrt{E / \varrho}\right)$, $\mathcal{O}\left(L^{-2} \sqrt{E I / \varrho A}\right)$ and $\mathcal{O}\left(L^{-1} \sqrt{G / \varrho}\right)$, respectively. From physical intuition, shearing should have the highest frequencies. We show that this is the case, constructing a pure 'shearing solution' of the linearised version of $(8)$ with frequency $\mathcal{O}(\sqrt{\kappa G A / \varrho I})$. To that end, we make the separation ansatz

$$
x=x(s)=s k, \quad p=p(t)=\cos \frac{\theta(t)}{2}+j \sin \frac{\theta(t)}{2}, \quad(s, t) \in[0, L] \times[0, T]
$$

with an unknown function $\theta=\theta(t), t \in[0, T]$. That is, we fix the centerline in straight position throughout the time interval $[0, T]$, and we assume a constant Euclidean shearing angle $\theta(t)$ along the whole rod $[0, L]$ at time $t$. Inserting (28) into (8), noting that $F=C_{\mathrm{SE}} \Gamma$ and $M=C_{\mathrm{BT}} K$, we receive the ordinary differential equation

$$
\ddot{\theta}=-\frac{\kappa G A}{\varrho I} \sin \theta \cos \theta \approx-\frac{\kappa G A}{\varrho I} \theta \quad \text { for }|\theta| \ll 1,
$$

its linearised version being the known linear oscillator equation. This yields the assertion, which is confirmed by numerical experiments.

Figure 6 displays typical stepsizes in explicit time integration, which we use to measure stiffness experimentally. It reveals, what we expect. The inextensible Kirchhoff model (low bending and torsional frequencies) is solved with the largest stepsizes. The extensible Kirchhoff model (additionally large extensional frequencies) is solved with medium stepsizes. The Cosserat model (additionally extremely high shearing frequencies) is necessarily solved with extremely small stepsizes by the explicit integrator. Note that the stiffness ratio between the extensible and inextensible Kirchhoff models is small. The reason is that the torsional eigenfrequencies are just slightly smaller than the extensional ones. Interestingly, the stepsizes for the Cosserat model do not depend on $N$, similarly as the shearing frequency obtained from (29) does not depend on $L$.

We point out that the Cosserat model is very stiff even for soft materials, such as rubber. Figure 7 exposes the stepsize behaviour for several solvers and the damped swinging rubber Cosserat rod from the same setup as in Figures 2, 3 and 5. Is demonstrates that explicit time integration is disastrous: Since the Jacobian contains very large eigenvalues with similar magnitude as in the undamped case, explicit solvers run on their stability limit, whereas implicit solution techniques behave fine, if strong damping is imposed on shearing and extension. The time steps of explicit solvers are nearly constant, whereas the stepsize patterns of implicit solvers nicely reflect the solution. The Cosserat model cannot be solved (half-)explicitly in acceptable computational time. Implicit time integration makes the efficient implementation of the analytical Jacobian unavoidable. Figure 4 (c) displays the structure of the latter in absolute coordinate formulation $(\dot{q}, \dot{v})=\Phi(q, v, t)$. The partial Jacobians $\partial \dot{v} / \partial q$ and $\partial \dot{v} / \partial v$ are banded with upper and lower bandwidth equal to $m=10$. Block banded Gaussian $L U$ factorisation [19] with complexity $\mathcal{O}\left(m^{2} N\right)$ is suitable for the treatment of the linear algebra involved in the corrector iterations.

Note that, since $I=\mathcal{O}\left(r^{4}\right)$ and $A=\mathcal{O}\left(r^{2}\right)$ for $r \rightarrow 0$, where $r>0$ is a typical cross section radius or diameter, we have $A / I=\mathcal{O}\left(1 / r^{2}\right)$. In all the rubber rod examples in this article, $E=5.0 \cdot 10^{6} \mathrm{Nm}^{-2}, G=1.67 \cdot 10^{6} \mathrm{Nm}^{-2}, r=5.0 \cdot 10^{-3} \mathrm{~m}, \varrho=1.1 \cdot 10^{3} \mathrm{~kg} \mathrm{~m}^{-3}, L=1.0 \mathrm{~m}$ with circular cross section, i. e. $\kappa=0.89, A=\pi r^{2}, I=\frac{1}{4} \pi r^{4}$, so that the eigenfrequencies for extension, bending, torsion and shearing are of orders $67.42 \mathrm{~s}^{-1}, 0.17 \mathrm{~s}^{-1}, 38.92 \mathrm{~s}^{-1}$ and $14683 \mathrm{~s}^{-1}$ respectively in the undamped case. Here we have shearing oscillations with very high frequencies - and usually very small amplitudes. If stiff components are not really necessary, one should eliminate them from the model, of course. 




Figure 7: Time stepsizes of different solvers for a damped rubber Cosserat rod. (ABSTOL $=$ REL$\mathrm{TOL}=1.0 \mathrm{E}^{-3}$ )

The question, which of the three classical rod models to choose, crucially depends on, what one wants to know. If the effect of shear is of subordinate importance, the Cosserat is not at all appropriate for high-performance or real-time computations, e. g. the simulation of cables.

\section{Conclusions}

The deformation of nonlinear rods was studied by geometrically exact models of increasing complexity. The Cosserat model considers shearing effects that result in very stiff model equations which can not be solved efficiently by (half-)explicit time integration methods. In the time integration by implicit methods, the efficiency is substantially improved exploiting the block band structure of the Jacobian in the semi-discretised system and using the analytically given parametrisation of the null space of the constraint matrix for constraints resulting from the normalisation of quaternions, which are used to parametrise the rotational degrees of freedom.

In the Kirchhoff model, the shearing effects are neglected and additional, more complex constraints have to be considered at each midpoint of the spatial grid. The differential-algebraic model equations are solved combining half-explicit integrators for the index-1 formulation with projection steps to avoid the drift-off effect and exploiting again the sparsity structure of the mass and constraint matrix. The additional negligence of rod extension in the inextensible Kirchhoff model allows a further speed-up of time integration but may result in kinematic singularities.

Future work will focus on an optimal representation of the null space for the constraint matrix in Kirchhoff models that would help to implement efficiently half-explicit methods for the index-2 formulation of the — non-stiff or mildly stiff — model equations.

Acknowledgements. We want to thank Joachim Linn (Fraunhofer ITWM Kaiserslautern) for many extensive and fruitful discussions. 


\section{References}

[1] Antman S.S. The theory of rods. Springer, 1972.

[2] Antman S.S. Dynamical problems for geometrically exact theories of nonlinearly viscoelastic rods. Journal of nonlinear science, Vol. 6, pp. 1-18, 1996.

[3] Antman S.S. Nonlinear problems of elasticity. Springer, 2005.

[4] Arnold M. Numerical methods for simulation in applied mechanics. In: Simulation techniques for applied mechanics, Eds: Arnold M. and Schiehlen W., Springer, pp. 191-246, 2008.

[5] Bauchau O. A., Epple A., Bottasso C. L. Scaling of constraints and augmented Lagrangian formulations in multibody dynamics simulations. Journal of computational and nonlinear dynamics, Vol. 4, No. 2, 2009.

[6] Bauchau O. A., Trainelli L. The vectorial parametrization of rotation. Nonlinear dynamics, Vol. 32 , No. 1, pp. $71-92,2003$.

[7] Bergou M., Wardetzky M., Robinson S., Audoly B., Grinspun E.: Discrete elastic rods. ACM transaction on graphics, Vol. 27, No. 3, SIGGRAPH, 2008.

[8] Betsch P., Leyendecker S. The discrete null space method for the energy consistent integration of constrained mechanical systems. Part II: Multibody dynamics. International journal for numerical methods in engineering, Vol. 67, pp. 499-552, 2006.

[9] Betsch P., Steinmann P. Frame-indifferent beam finite elements based upon the geometrically exact beam theory. International journal for numerical methods in engineering, Vol. 54, pp. 1755-1788, 2002.

[10] Bobenko A.I., Suris Y.B. Discrete time Lagrangian mechanics on Lie groups with an application to the Lagrange top. Communications in mathematical physics, Vol. 204, pp. 147-188, 1999.

[11] Cardona A., Géradin M. Flexible multibody dynamics. A finite element approach. Wiley, 2001.

[12] Cartan H. Formes différentielles - Applications élémentaires au calcul des variations et à la théorie des courbes et des surfaces. Hermann Paris, 1967, transl.: Differential Forms, Dover, 2006.

[13] Craig R. R. jr., Kurdila A. J. Fundamentals of structural mechanics. Wiley, 2006.

[14] Crisfield M. A., Jelenic G. Objectivity of strain measures in the geometrically exact three-dimensional beam theory and its finite element implementation. Proceedings of the royal society London, Vol. 455, pp. 1125-1147, 1999.

[15] Dill E.H. Kirchhoff's theory of rods. Archives for history of exact sciences, Vol. 44, No. 1, pp. 1-23, 1992.

[16] Ebbinghaus H. D. et al. Numbers. Springer, 1992.

[17] Eich-Soellner E., Führer C. Numerical methods in multibody dynamics. Teubner, 1998.

[18] Gear C. W. Maintaining solution invariants in the numerical solution of ODEs. SIAM journal on scientific and statistical computing, Vol. 7, pp. 734-743, 1986.

[19] Golub G., Van Loan C. Matrix computations. Third edition, The John Hopkins university press, 1996.

[20] Hanson A. J. Visualizing quaternions. Elsevier, 2005.

[21] Hodges D. H. Nonlinear composite beam theory. Progress in astronautics and aeronautics, Vol. $213,2006$.

[22] Hairer E., Wanner G. Solving ordinary differential equations II. Springer, 1996.

[23] Ibrahimbegović A. On finite element implementations of geometrically nonlinear Reissner's beam theory: Three dimensional curved beam elements. Computer methods in applied mechanics and engineering, Vol. 112, pp. 11-26, 1995.

[24] Jung P. A discrete mechanics approach to Cosserat rod theory - static equilibria. Diploma thesis, TU Kaiserslautern, 2009.

[25] Landau L. D., Lifshitz E. M. Theory of elasticity. Course of theoretical physics, Vol. 7, 3rd edition, Butterworth Heinemann, 1986.

[26] Lang H., Linn J. Lagrangian field theory in space-time for geometrically exact Cosserat rods. Report of the Fraunhofer ITWM, Vol. 150, 2009.

[27] Lang H., Linn J., Arnold M. Multibody dynamics simulation of geometrically exact Cosserat rods. Multibody 2009, ECCOMAS thematic conference, June 29 - July 2, Warsaw, Poland, available as well as Report of the Fraunhofer ITWM, Vol. 159, 2009.

[28] Love A. E. H. Treatise on the mathematical theory of elasticity. Dover, 1944.

[29] Richtmyer R.D., Morton K. W. Difference methods for initial value problems. Interscience publishers, New York, 1967. 
[30] Podio-Guidugli P. A primer in elasticity. Journal of elasticity, Vol. 58, No. 1, pp. 1-104, 2000.

[31] Romero I. The interpolation of rotations and its application to finite-element models of geometrically exact rods. Computational mechanics, Vol. 34, pp. 121-133, 2004.

[32] Sander O. Geodesic finite elements for Cosserat rods. Preprint, Freie Universität Berlin, 2009.

[33] Schiehlen W., Eberhard P. Technische Dynamik. Modelle für Regelung und Simulation. Teubner, 2004.

[34] Schwab A. L., Meijaard P. J. How to draw Euler angles and utilize Euler parameters. Proceedings of IDETC/CIE, 2008.

[35] Schwab A. L., Meijaard P. J. Beam benchmark problems for validation of flexible multibody dynamics codes. Multibody 2009, ECCOMAS thematic conference, June 29 - July 2, Warsaw, Poland, 2009.

[36] Shabana A. A. Dynamics of multibody systems. Cambridge, 2005.

[37] Shampine L.F., Reichelt M. W.: The Matlab ODE suite. SIAM journal on scientific computing, Vol. 18, No. 1, 1997.

[38] Simo J. C. A finite strain beam formulation. The three dimensional dynamic problem. Part I. Computer methods in applied mechanics and engineering, Vol. 49, pp. 55-70, 1985.

[39] Simo J. C., Vu-Quoc L. On the dynamics in space of rods undergoing large motions - A geometrically exact approach. Computer methods in applied mechanics and engineering, Vol. 66, pp. 125-161, 1988.

[40] Spillmann J., Teschner M. CoRdE. Cosserat rod elements for the dynamic simulation of one-dimensional elastic objects. Eurographics/ACM SIGGRAPH, pp. 1-10, 2007. 


\section{Published reports of the Fraunhofer ITWM}

The PDF-files of the following reports are available under:

\section{www.itwm.fraunhofer.de/de/ zentral_berichte/berichte}

1. D. Hietel, K. Steiner, J. Struckmeier A Finite - Volume Particle Method for Compressible Flows (19 pages, 1998)

2. M. Feldmann, S. Seibold

Damage Diagnosis of Rotors: Application of Hilbert Transform and Multi-Hypothesis Testing

Keywords: Hilbert transform, damage diagnosis,

Kalman filtering, non-linear dynamics

(23 pages, 1998)

3. Y. Ben-Haim, S. Seibold

Robust Reliability of Diagnostic MultiHypothesis Algorithms: Application to Rotating Machinery

Keywords: Robust reliability, convex models, Kalman filtering, multi-hypothesis diagnosis, rotating machinery, crack diagnosis

(24 pages, 1998)

\section{F.-Th. Lentes, N. Siedow}

Three-dimensional Radiative Heat Transfer in Glass Cooling Processes

(23 pages, 1998)

5. A. Klar, R. Wegener

A hierarchy of models for multilane vehicular traffic

Part I: Modeling

(23 pages, 1998)

Part II: Numerical and stochastic investigations (17 pages, 1998)

\section{A. Klar, N. Siedow}

Boundary Layers and Domain Decomposition for Radiative Heat Transfer and Diffusion Equations: Applications to Glass Manufacturing Processes

(24 pages, 1998)

7. I. Choquet

Heterogeneous catalysis modelling and numerical simulation in rarified gas flows Part l: Coverage locally at equilibrium (24 pages, 1998)

8. J. Ohser, B. Steinbach, C. Lang Efficient Texture Analysis of Binary Images (17 pages, 1998)

9. J. Orlik

Homogenization for viscoelasticity of the integral type with aging and shrinkage (20 pages, 1998)

10. J. Mohring

Helmholtz Resonators with Large Aperture (21 pages, 1998)
11. H. W. Hamacher, A. Schöbel On Center Cycles in Grid Graphs (15 pages, 1998)

12. H. W. Hamacher, K.-H. Küfer Inverse radiation therapy planning a multiple objective optimisation approach (14 pages, 1999)

13. C. Lang, J. Ohser, R. Hilfer On the Analysis of Spatial Binary Images (20 pages, 1999)

14. M. Junk

On the Construction of Discrete Equilibrium Distributions for Kinetic Schemes (24 pages, 1999)

15. M. Junk, S. V. Raghurame Rao

A new discrete velocity method for NavierStokes equations

(20 pages, 1999)

16. H. Neunzert

Mathematics as a Key to Key Technologies (39 pages (4 PDF-Files), 1999)

17. J. Ohser, K. Sandau

Considerations about the Estimation of the Size Distribution in Wicksell's Corpuscle Problem

(18 pages, 1999)

18. E. Carrizosa, H. W. Hamacher, R. Klein, S. Nickel

Solving nonconvex planar location problems by finite dominating sets

Keywords: Continuous Location, Polyhedral Gauges, Finite Dominating Sets, Approximation, Sandwich Algorithm, Greedy Algorithm

(19 pages, 2000)

\section{A. Becker}

A Review on Image Distortion Measures Keywords: Distortion measure, human visual system (26 pages, 2000)

20. H. W. Hamacher, M. Labbé, S. Nickel, T. Sonneborn

Polyhedral Properties of the Uncapacitated Multiple Allocation Hub Location Problem Keywords: integer programming, hub location, facility location, valid inequalities, facets, branch and cut (21 pages, 2000)

21. H. W. Hamacher, A. Schöbel

Design of Zone Tariff Systems in Public Transportation

(30 pages, 2001)

22. D. Hietel, M. Junk, R. Keck, D. Teleaga The Finite-Volume-Particle Method for Conservation Laws (16 pages, 2001)

23. T. Bender, H. Hennes, J. Kalcsics, M. T. Melo, S. Nickel

Location Software and Interface with GIS and Supply Chain Management Keywords: facility location, software development, geographical information systems, supply chain management

(48 pages, 2001)
24. H. W. Hamacher, S. A. Tjandra Mathematical Modelling of Evacuation Problems: A State of Art (44 pages, 2001)

25. J. Kuhnert, S. Tiwari

Grid free method for solving the Poisson equation

Keywords: Poisson equation, Least squares method, Grid free method

(19 pages, 2001)

26. T. Götz, H. Rave, D. Reinel-Bitzer, K. Steiner, $\mathrm{H}$. Tiemeier

Simulation of the fiber spinning process Keywords: Melt spinning, fiber model, Lattice Boltzmann, CFD

(19 pages, 2001)

\section{A. Zemitis}

On interaction of a liquid film with an obstacle Keywords: impinging jets, liquid film, models, numerical solution, shape

22 pages, 2001)

\section{I. Ginzburg, K. Steiner}

Free surface lattice-Boltzmann method to model the filling of expanding cavities by Bingham Fluids

Keywords: Generalized LBE, free-surface phenomena interface boundary conditions, filling processes, Bing ham viscoplastic model, regularized models

(22 pages, 2001)

\section{H. Neunzert}

"Denn nichts ist für den Menschen als Menschen etwas wert, was er nicht mit Leidenschaft tun kann

Vortrag anlässlich der Verleihung des Akademiepreises des Landes RheinlandPfalz am 21.11.2001

Keywords: Lehre, Forschung, angewandte Mathematik, Mehrskalenanalyse, Strömungsmechanik (18 pages, 2001)

30. J. Kuhnert, S. Tiwari

Finite pointset method based on the projection method for simulations of the incompressible Navier-Stokes equations

Keywords: Incompressible Navier-Stokes equations, Meshfree method, Projection method, Particle scheme, Least squares approximation

AMS subject classification: 76D05, 76M28

(25 pages, 2001)

31. R. Korn, M. Krekel

Optimal Portfolios with Fixed Consumption or Income Streams

Keywords: Portfolio optimisation, stochastic control, HJB equation, discretisation of control problems (23 pages, 2002)

32. M. Krekel

Optimal portfolios with a loan dependent credit spread

Keywords: Portfolio optimisation, stochastic control, HJB equation, credit spread, log utility, power utility, non-linear wealth dynamics

(25 pages, 2002)

33. J. Ohser, W. Nagel, K. Schladitz

The Euler number of discretized sets - on the choice of adjacency in homogeneous lattices Keywords: image analysis, Euler number, neighborhod relationships, cuboidal lattice

(32 pages, 2002) 
34. I. Ginzburg, K. Steiner

Lattice Boltzmann Model for Free-Surface flow and Its Application to Filling Process in Casting

Keywords: Lattice Boltzmann models; free-surface phenomena; interface boundary conditions; filling processes; injection molding; volume of fluid method; interface boundary conditions; advection-schemes; upwind-schemes

(54 pages, 2002)

35. M. Günther, A. Klar, T. Materne, R. Wegener

Multivalued fundamental diagrams and stop and go waves for continuum traffic equations Keywords: traffic flow, macroscopic equations, kinetic derivation, multivalued fundamental diagram, stop and go waves, phase transitions

(25 pages, 2002)

36. S. Feldmann, P. Lang, D. Prätzel-Wolters Parameter influence on the zeros of network determinants

Keywords: Networks, Equicofactor matrix polynomials, Realization theory, Matrix perturbation theory

(30 pages, 2002)

\section{K. Koch, J. Ohser, K. Schladitz} Spectral theory for random closed sets and estimating the covariance via frequency space

Keywords: Random set, Bartlett spectrum, fast Fourier transform, power spectrum

(28 pages, 2002)

\section{D. d'Humières, I. Ginzburg}

Multi-reflection boundary conditions for lattice Boltzmann models

Keywords: lattice Boltzmann equation, boudary condistions, bounce-back rule, Navier-Stokes equation

(72 pages, 2002)

\section{R. Korn}

\section{Elementare Finanzmathematik}

Keywords: Finanzmathematik, Aktien, Optionen, Portfolio-Optimierung, Börse, Lehrerweiterbildung, Mathematikunterricht

(98 pages, 2002)

40. J. Kallrath, M. C. Müller, S. Nickel

Batch Presorting Problems:

Models and Complexity Results

Keywords: Complexity theory, Integer programming,

Assigment, Logistics

(19 pages, 2002)

\section{J. Linn}

On the frame-invariant description of the phase space of the Folgar-Tucker equation Key words: fiber orientation, Folgar-Tucker equation, injection molding

(5 pages, 2003)

\section{T. Hanne, S. Nickel}

A Multi-Objective Evolutionary Algorithm for Scheduling and Inspection Planning in Software Development Projects

Key words: multiple objective programming, project management and scheduling, software development, evolutionary algorithms, efficient set

(29 pages, 2003)

43. T. Bortfeld, K.-H. Küfer, M. Monz,

A. Scherrer, C. Thieke, H. Trinkaus

Intensity-Modulated Radiotherapy - A Large Scale Multi-Criteria Programming Problem
Keywords: multiple criteria optimization, representative systems of Pareto solutions, adaptive triangulation, clustering and disaggregation techniques, visualization of Pareto solutions, medical physics, external beam radiotherapy planning, intensity modulated radiotherapy (31 pages, 2003)

\section{T. Halfmann, T. Wichmann}

Overview of Symbolic Methods in Industrial Analog Circuit Design

Keywords: $C A D$, automated analog circuit design, sym bolic analysis, computer algebra, behavioral modeling, system simulation, circuit sizing, macro modeling, differential-algebraic equations, index

(17 pages, 2003)

\section{S. E. Mikhailov, J. Orlik}

Asymptotic Homogenisation in Strength and Fatigue Durability Analysis of Composites

Keywords: multiscale structures, asymptotic homogenization, strength, fatigue, singularity, non-local conditions

(14 pages, 2003)

46. P. Domínguez-Marín, P. Hansen, N. Mladenovi'c , S. Nickel

Heuristic Procedures for Solving the Discrete Ordered Median Problem

Keywords: genetic algorithms, variable neighborhood search, discrete facility location

(31 pages, 2003)

47. N. Boland, P. Domínguez-Marín, S. Nickel, J. Puerto

Exact Procedures for Solving the Discrete Ordered Median Problem

Keywords: discrete location, Integer programming

(41 pages, 2003)

48. S. Feldmann, P. Lang

Padé-like reduction of stable discrete linear systems preserving their stability Keywords: Discrete linear systems, model reduction, stability, Hankel matrix, Stein equation

(16 pages, 2003)

49. J. Kallrath, S. Nickel

A Polynomial Case of the Batch Presorting Problem

Keywords: batch presorting problem, online optimization, competetive analysis, polynomial algorithms, logistics (17 pages, 2003)

\section{T. Hanne, H. L. Trinkaus}

knowCube for MCDM -

Visual and Interactive Support for Multicriteria Decision Making

Key words: Multicriteria decision making, knowledge management, decision support systems, visual interfaces, interactive navigation, real-life applications. (26 pages, 2003)

51. O. lliev, V. Laptev

On Numerical Simulation of Flow Through Oil Filters

Keywords: oil filters, coupled flow in plain and porous media, Navier-Stokes, Brinkman, numerical simulation (8 pages, 2003)

52. W. Dörfler, O. Iliev, D. Stoyanov, D. Vassileva On a Multigrid Adaptive Refinement Solver for Saturated Non-Newtonian Flow in Porous Media

Keywords: Nonlinear multigrid, adaptive refinement, non-Newtonian flow in porous media

(17 pages, 2003)
53. S. Kruse

On the Pricing of Forward Starting Options under Stochastic Volatility

Keywords: Option pricing, forward starting options, Heston model, stochastic volatility, cliquet options (11 pages, 2003)

54. O. Iliev, D. Stoyanov

Multigrid - adaptive local refinement solver for incompressible flows

Keywords: Navier-Stokes equations, incompressible flow projection-type splitting, SIMPLE, multigrid methods, adaptive local refinement, lid-driven flow in a cavity (37 pages, 2003)

\section{V. Starikovicius}

The multiphase flow and heat transfer in porous media

Keywords: Two-phase flow in porous media, various formulations, global pressure, multiphase mixture model, numerical simulation

(30 pages, 2003)

\section{P. Lang, A. Sarishvili, A. Wirsen}

Blocked neural networks for knowledge extraction in the software development process Keywords: Blocked Neural Networks, Nonlinear Regression, Knowledge Extraction, Code Inspection (21 pages, 2003)

\section{H. Knaf, P. Lang, S. Zeiser}

Diagnosis aiding in Regulation

Thermography using Fuzzy Logic

Keywords: fuzzy logic, knowledge representation, expert system

(22 pages, 2003)

58. M. T. Melo, S. Nickel, F. Saldanha da Gama Largescale models for dynamic multicommodity capacitated facility location Keywords: supply chain management, strategic planning, dynamic location, modeling (40 pages, 2003)

\section{J. Orlik}

Homogenization for contact problems with periodically rough surfaces

Keywords: asymptotic homogenization, contact problems (28 pages, 2004)

60. A. Scherrer, K.-H. Küfer, M. Monz,

F. Alonso, T. Bortfeld

IMRT planning on adaptive volume structures - a significant advance of computational complexity

Keywords: Intensity-modulated radiation therapy (IMRT), inverse treatment planning, adaptive volume structures, hierarchical clustering, local refinement, adaptive clustering, convex programming, mesh generation, multi-grid methods

(24 pages, 2004)

\section{D. Kehrwald}

Parallel lattice Boltzmann simulation of complex flows

Keywords: Lattice Boltzmann methods, parallel computing, microstructure simulation, virtual material design, pseudo-plastic fluids, liquid composite moulding (12 pages, 2004)

62. O. Iliev, J. Linn, M. Moog, D. Niedziela, V. Starikovicius

On the Performance of Certain Iterative Solvers for Coupled Systems Arising in Discretization of Non-Newtonian Flow Equations 
Keywords: Performance of iterative solvers, Preconditioners, Non-Newtonian flow

(17 pages, 2004)

\section{R. Ciegis, O. Iliev, S. Rief, K. Steiner}

On Modelling and Simulation of Different Regimes for Liquid Polymer Moulding Keywords: Liquid Polymer Moulding, Modelling, Simulation, Infiltration, Front Propagation, non-Newtonian flow in porous media

(43 pages, 2004)

\section{T. Hanne, H. Neu}

Simulating Human Resources in

\section{Software Development Processes}

Keywords: Human resource modeling, software process, productivity, human factors, learning curve

(14 pages, 2004)

\section{O. Iliev, A. Mikelic, P. Popov}

Fluid structure interaction problems in de-

formable porous media: Toward permeability of deformable porous media

Keywords: fluid-structure interaction, deformable porous media, upscaling, linear elasticity, stokes, finite elements

(28 pages, 2004)

66. F. Gaspar, O. Iliev, F. Lisbona, A. Naumovich, P. Vabishchevich

On numerical solution of 1-D poroelasticity equations in a multilayered domain Keywords: poroelasticity, multilayered material, finite volume discretization, MAC type grid

(41 pages, 2004)

67. J. Ohser, K. Schladitz, K. Koch, M. Nöthe Diffraction by image processing and its application in materials science

Keywords: porous microstructure, image analysis, random set, fast Fourier transform, power spectrum, Bartlett spectrum

(13 pages, 2004)

\section{H. Neunzert}

\section{Mathematics as a Technology: Challenges} for the next 10 Years

Keywords: applied mathematics, technology, modelling, simulation, visualization, optimization, glass processing, spinning processes, fiber-fluid interaction, trubulence effects, topological optimization, multicriteria optimiza tion, Uncertainty and Risk, financial mathematics, Malliavin calculus, Monte-Carlo methods, virtual material design, filtration, bio-informatics, system biology

(29 pages, 2004)

69. R. Ewing, O. lliev, R. Lazarov, A. Naumovich On convergence of certain finite difference discretizations for 1D poroelasticity interface problems

Keywords: poroelasticity, multilayered material, finite volume discretizations, MAC type grid, error estimates (26 pages, 2004 )

70. W. Dörfler, O. Iliev, D. Stoyanov, D. Vassileva On Efficient Simulation of Non-Newtonian Flow in Saturated Porous Media with a Multigrid Adaptive Refinement Solver Keywords: Nonlinear multigrid, adaptive renement non-Newtonian in porous media (25 pages, 2004)

\section{J. Kalcsics, S. Nickel, M. Schröde}

Towards a Unified Territory Design Approach - Applications, Algorithms and GIS Integration Keywords: territory desgin, political districting, sales territory alignment, optimization algorithms, Geographical Information Systems

(40 pages, 2005)
72. K. Schladitz, S Peters, D Reinel-Bitzer, A. Wiegmann, J. Ohser

Design of acoustic trim based on geometric modeling and flow simulation for non-woven Keywords: random system of fibers, Poisson line process, flow resistivity, acoustic absorption, Lattice-Boltzmann method, non-woven (21 pages, 2005)

\section{V. Rutka, A. Wiegmann}

Explicit Jump Immersed Interface Method for virtual material design of the effective elastic moduli of composite materials Keywords: virtual material design, explicit jump immersed interface method, effective elastic moduli, composite materials

(22 pages, 2005)

\section{T. Hanne}

Eine Übersicht zum Scheduling von Baustellen Keywords: Projektplanung, Scheduling, Bauplanung, Bauindustrie

(32 pages, 2005)

75. J. Linn

The Folgar-Tucker Model as a Differetial Algebraic System for Fiber Orientation Calculation

Keywords: fiber orientation, Folgar-Tucker model, invariants, algebraic constraints, phase space, trace stability

(15 pages, 2005)

76. M. Speckert, K. Dreßler, H. Mauch, A. Lion, G. J. Wierda

Simulation eines neuartigen Prüfsystems für Achserprobungen durch MKS-Modellierung einschließlich Regelung Keywords: virtual test rig, suspension testing, multibody simulation, modeling hexapod test rig, opti mization of test rig configuration

(20 pages, 2005)

77. K.-H. Küfer, M. Monz, A. Scherrer, P. Süss, F. Alonso, A. S. A. Sultan, Th. Bortfeld,

D. Craft, Chr. Thieke

Multicriteria optimization in intensity modulated radiotherapy planning Keywords: multicriteria optimization, extreme solutions, real-time decision making, adaptive approxima tion schemes, clustering methods, IMRT planning, reverse engineering

(51 pages, 2005)

78. S. Amstutz, H. Andrä

A new algorithm for topology optimization using a level-set method

Keywords: shape optimization, topology optimization topological sensitivity, level-set

(22 pages, 2005)

\section{N. Ettrich}

Generation of surface elevation models for urban drainage simulation

Keywords: Flooding, simulation, urban elevation models, laser scanning

(22 pages, 2005

80. H. Andrä, J. Linn, I. Matei, I. Shklyar, K. Steiner, E. Teichmann

OPTCAST - Entwicklung adäquater Struk turoptimierungsverfahren für Gießereien Technischer Bericht (KURZFASSUNG) Keywords: Topologieoptimierung, Level-Set-Methode Gießprozesssimulation, Gießtechnische Restriktionen, CAE-Kette zur Strukturoptimierung

(77 pages, 2005)
81. N. Marheineke, R. Wegener Fiber Dynamics in Turbulent Flows Part I: General Modeling Framework Keywords: fiber-fluid interaction; Cosserat rod; turbuence modeling: Kolmogorov's energy spectrum; double-velocity correlations; differentiable Gaussian fields (20 pages, 2005)

\section{Part II: Specific Taylor Drag}

Keywords: flexible fibers; $k-\varepsilon$ turbulence model; fiber-turbulence interaction scales; air drag; random Gaussian aerodynamic force; white noise; stochastic differential equations; ARMA process

(18 pages, 2005)

\section{C. H. Lampert, O. Wirjadi}

An Optimal Non-Orthogonal Separation of the Anisotropic Gaussian Convolution Filter Keywords: Anisotropic Gaussian filter, linear filtering, or entation space, $n D$ image processing, separable filters (25 pages, 2005)

83. H. Andrä, D. Stoyanov

Error indicators in the parallel finite element solver for linear elasticity DDFEM Keywords: linear elasticity, finite element method, hierarchical shape functions, domain decom-position, parallel implementation, a posteriori error estimates (21 pages, 2006)

84. M. Schröder, I. Solchenbach Optimization of Transfer Quality in Regional Public Transit

Keywords: public transit, transfer quality, quadratic assignment problem

(16 pages, 2006

85. A. Naumovich, F. J. Gaspar

On a multigrid solver for the three-dimensional Biot poroelasticity system in multilayered domains

Keywords: poroelasticity, interface problem, multigrid, operator-dependent prolongation

(11 pages, 2006)

86. S. Panda, R. Wegener, N. Marheineke Slender Body Theory for the Dynamics of Curved Viscous Fibers

Keywords: curved viscous fibers; fluid dynamics; NavierStokes equations; free boundary value problem; asymptotic expansions; slender body theory

(14 pages, 2006)

87. E. Ivanov, H. Andrä, A. Kudryavtsev Domain Decomposition Approach for Automatic Parallel Generation of Tetrahedral Grids Key words: Grid Generation, Unstructured Grid, Delau nay Triangulation, Parallel Programming, Domain Decomposition, Load Balancing

(18 pages, 2006)

88. S. Tiwari, S. Antonov, D. Hietel, J. Kuhnert, R. Wegener

A Meshfree Method for Simulations of In teractions between Fluids and Flexible Structures

Key words: Meshfree Method, FPM, Fluid Structure Interaction, Sheet of Paper, Dynamical Coupling (16 pages, 2006)

89. R. Ciegis, O. Iliev, V. Starikovicius, K. Steine Numerical Algorithms for Solving Problems of Multiphase Flows in Porous Media Keywords: nonlinear algorithms, finite-volume method software tools, porous media, flows

16 pages, 2006) 
90. D. Niedziela, O. Iliev, A. Latz

On 3D Numerical Simulations of Viscoelastic Fluids

Keywords: non-Newtonian fluids, anisotropic viscosity, integral constitutive equation

(18 pages, 2006)

\section{A. Winterfeld}

Application of general semi-infinite Programming to Lapidary Cutting Problems Keywords: large scale optimization, nonlinear programming, general semi-infinite optimization, design centering, clustering

(26 pages, 2006)

\section{J. Orlik, A. Ostrovska}

Space-Time Finite Element Approximation and Numerical Solution of Hereditary Linear Viscoelasticity Problems

Keywords: hereditary viscoelasticity; kern approximation by interpolation; space-time finite element approximation, stability and a priori estimate

(24 pages, 2006)

93. V. Rutka, A. Wiegmann, H. Andrä EJIIM for Calculation of effective Elastic Moduli in 3D Linear Elasticity

Keywords: Elliptic PDE, linear elasticity, irregular domain, finite differences, fast solvers, effective elastic moduli

(24 pages, 2006)

\section{A. Wiegmann, A. Zemitis}

EJ-HEAT: A Fast Explicit Jump Harmonic Averaging Solver for the Effective Heat Conductivity of Composite Materials Keywords: Stationary heat equation, effective thermal conductivity, explicit jump, discontinuous coefficients, virtual material design, microstructure simulation, EJ-HEAT

(21 pages, 2006)

\section{A. Naumovich}

On a finite volume discretization of the three-dimensional Biot poroelasticity system in multilayered domains

Keywords: Biot poroelasticity system, interface problems, finite volume discretization, finite difference method (21 pages, 2006)

\section{M. Krekel, J. Wenzel}

A unified approach to Credit Default Swaption and Constant Maturity Credit Default Swap valuation

Keywords: LIBOR market model, credit risk, Credit Default Swaption, Constant Maturity Credit Default Swapmethod

(43 pages, 2006)

\section{A. Dreyer}

\section{Interval Methods for Analog Circiuts}

Keywords: interval arithmetic, analog circuits, tolerance analysis, parametric linear systems, frequency response, symbolic analysis, CAD, computer algebra

(36 pages, 2006)

98. N. Weigel, S. Weihe, G. Bitsch, K. Dreßler Usage of Simulation for Design and Optimization of Testing

Keywords: Vehicle test rigs, MBS, control, hydraulics,

testing philosophy

(14 pages, 2006)

99. H. Lang, G. Bitsch, K. Dreßler, M. Speckert Comparison of the solutions of the elastic and elastoplastic boundary value problems
Keywords: Elastic BVP, elastoplastic BVP, variational inequalities, rate-independency, hysteresis, linear kinematic hardening, stop- and play-operator

(21 pages, 2006)

100. M. Speckert, K. Dreßler, H. Mauch MBS Simulation of a hexapod based suspension test rig

Keywords: Test rig, MBS simulation, suspension,

hydraulics, controlling, design optimization

(12 pages, 2006)

101. S. Azizi Sultan, K.-H. Küfer

A dynamic algorithm for beam orientations in multicriteria IMRT planning

Keywords: radiotherapy planning, beam orientation optimization, dynamic approach, evolutionary algorithm, global optimization

(14 pages, 2006)

102. T. Götz, A. Klar, N. Marheineke, R. Wegener A Stochastic Model for the Fiber Lay-down Process in the Nonwoven Production Keywords: fiber dynamics, stochastic Hamiltonian system, stochastic averaging

(17 pages, 2006)

103. Ph. Süss, K.-H. Küfer

Balancing control and simplicity: a variable aggregation method in intensity modulated radiation therapy planning

Keywords: IMRT planning, variable aggregation, clustering methods

(22 pages, 2006)

104. A. Beaudry, G. Laporte, T. Melo, S. Nickel Dynamic transportation of patients in hospitals

Keywords: in-house hospital transportation, dial-a-ride, dynamic mode, tabu search

(37 pages, 2006)

105. Th. Hanne

Applying multiobjective evolutionary algorithms in industrial projects

Keywords: multiobjective evolutionary algorithms, discrete optimization, continuous optimization, electronic circuit design, semi-infinite programming, scheduling (18 pages, 2006)

106. J. Franke, S. Halim

Wild bootstrap tests for comparing signals and images

Keywords: wild bootstrap test, texture classification, textile quality control, defect detection, kernel estimate, nonparametric regression

(13 pages, 2007)

107. Z. Drezner, S. Nickel

Solving the ordered one-median problem in the plane

Keywords: planar location, global optimization, ordered median, big triangle small triangle method, bounds, numerical experiments

(21 pages, 2007)

108. Th. Götz, A. Klar, A. Unterreiter, R. Wegener

Numerical evidance for the non-existing of solutions of the equations desribing rotational fiber spinning

Keywords: rotational fiber spinning, viscous fibers, boundary value problem, existence of solutions (11 pages, 2007)
109. Ph. Süss, K.-H. Küfer

Smooth intensity maps and the BortfeldBoyer sequencer

Keywords: probabilistic analysis, intensity modulated radiotherapy treatment (IMRT), IMRT plan application step-and-shoot sequencing

(8 pages, 2007)

110. E. Ivanov, O. Gluchshenko, H. Andrä, A. Kudryavtsev

Parallel software tool for decomposing and meshing of $3 d$ structures

Keywords: a-priori domain decomposition, unstructured grid, Delaunay mesh generation

(14 pages, 2007)

111. O. lliev, R. Lazarov, J. Willems Numerical study of two-grid preconditioners for 1d elliptic problems with highly oscillating discontinuous coefficients Keywords: two-grid algorithm, oscillating coefficients, preconditioner

(20 pages, 2007)

112. L. Bonilla, T. Götz, A. Klar, N. Marheineke, R. Wegener

Hydrodynamic limit of the Fokker-Planckequation describing fiber lay-down processes

Keywords: stochastic dierential equations, FokkerPlanck equation, asymptotic expansion, Ornstein-

Uhlenbeck process

(17 pages, 2007)

\section{S. Rief}

Modeling and simulation of the pressing section of a paper machine

Keywords: paper machine, computational fluid dynamics, porous media

(41 pages, 2007)

114. R. Ciegis, O. Iliev, Z. Lakdawala

On parallel numerical algorithms for simulating industrial filtration problems

Keywords: Navier-Stokes-Brinkmann equations, finite volume discretization method, SIMPLE, parallel computing, data decomposition method

(24 pages, 2007)

\section{N. Marheineke, R. Wegener}

Dynamics of curved viscous fibers with surface tension

Keywords: Slender body theory, curved viscous bers with surface tension, free boundary value problem (25 pages, 2007)

116. S. Feth, J. Franke, M. Speckert Resampling-Methoden zur mse-Korrektur und Anwendungen in der Betriebsfestigkeit Keywords: Weibull, Bootstrap, Maximum-Likelihood, Betriebsfestigkeit

(16 pages, 2007)

117. H. Knaf

Kernel Fisher discriminant functions - a concise and rigorous introduction

Keywords: wild bootstrap test, texture classification, textile quality control, defect detection, kernel estimate, nonparametric regression

(30 pages, 2007)

118. O. Iliev, I. Rybak

On numerical upscaling for flows in heterogeneous porous media 
Keywords: numerical upscaling heterogeneous porous media, single phase flow, Darcy's law, multiscale problem, effective permeability, multipoint flux approximation, anisotropy

(17 pages, 2007)

\section{O. Iliev, I. Rybak}

On approximation property of multipoint flux approximation method

Keywords: Multipoint flux approximation, finite volume method, elliptic equation, discontinuous tensor coeffi-

cients, anisotropy

(15 pages, 2007)

120. O. Iliev, I. Rybak, J. Willems

On upscaling heat conductivity for a class of industrial problems

Keywords: Multiscale problems, effective heat conductivity, numerical upscaling, domain decomposition

(21 pages, 2007)

121. R. Ewing, O. Iliev, R. Lazarov, I. Rybak On two-level preconditioners for flow in porous media

Keywords: Multiscale problem, Darcy's law, single phase flow, anisotropic heterogeneous porous media, numerical upscaling, multigrid, domain decomposition, efficient preconditioner

(18 pages, 2007)

122. M. Brickenstein, A. Dreyer

POLYBORI: A Gröbner basis framework for Boolean polynomials

Keywords: Gröbner basis, formal verification, Boolean polynomials, algebraic cryptoanalysis, satisfiability

(23 pages, 2007)

\section{O. Wirjad}

Survey of $3 d$ image segmentation methods Keywords: image processing, 3d, image segmentation, binarization

(20 pages, 2007)

\section{S. Zeytun, A. Gupta}

A Comparative Study of the Vasicek and the CIR Model of the Short Rate

Keywords: interest rates, Vasicek model, CIR-model,

calibration, parameter estimation

(17 pages, 2007)

\section{G. Hanselmann, A. Sarishvili}

Heterogeneous redundancy in software quality prediction using a hybrid Bayesian approach

Keywords: reliability prediction, fault prediction, nonhomogeneous poisson process, Bayesian model averaging

(17 pages, 2007)

126. V. Maag, M. Berger, A. Winterfeld, K.-H Küfer

A novel non-linear approach to minimal area rectangular packing

Keywords: rectangular packing, non-overlapping constraints, non-linear optimization, regularization, relaxation

(18 pages, 2007)

127. M. Monz, K.-H. Küfer, T. Bortfeld, C. Thieke Pareto navigation - systematic multi-criteria-based IMRT treatment plan determination

Keywords: convex, interactive multi-objective optimization, intensity modulated radiotherapy planning

(15 pages, 2007)
128. M. Krause A Scherrer

On the role of modeling parameters in IMRT plan optimization

Keywords: intensity-modulated radiotherapy (IMRT) inverse IMRT planning, convex optimization, sensitivity analysis, elasticity, modeling parameters, equivalent uniform dose (EUD)

(18 pages, 2007)

\section{A. Wiegmann}

Computation of the permeability of porous materials from their microstructure by FFFStokes

Keywords: permeability, numerical homogenization

fast Stokes solver

(24 pages, 2007)

130. T. Melo, S. Nickel, F. Saldanha da Gama Facility Location and Supply Chain Management - A comprehensive review

Keywords: facility location, supply chain management network design

(54 pages, 2007)

131. T. Hanne, T. Melo, S. Nickel

Bringing robustness to patient flow management through optimized patient transports in hospitals

Keywords: Dial-a-Ride problem, online problem, case study, tabu search, hospital logistics

(23 pages, 2007)

132. R. Ewing, O. Iliev, R. Lazarov, I. Rybak, J. Willems

An efficient approach for upscaling properties of composite materials with high contrast of coefficients

Keywords: effective heat conductivity, permeability of fractured porous media, numerical upscaling, fibrous insulation materials, metal foams

(16 pages, 2008)

133. S. Gelareh, S. Nickel

New approaches to hub location problems in public transport planning Keywords: integer programming, hub location, transportation, decomposition, heuristic

(25 pages, 2008)

134. G. Thömmes, J. Becker, M. Junk, A. K. Vaikuntam, D. Kehrwald, A. Klar, K. Steiner, A. Wiegmann

A Lattice Boltzmann Method for immiscible multiphase flow simulations using the Level Set Method

Keywords: Lattice Boltzmann method, Level Set method, free surface, multiphase flow (28 pages, 2008

\section{J. Orlik}

Homogenization in elasto-plasticity

Keywords: multiscale structures, asymptotic homogenization, nonlinear energy

(40 pages, 2008)

136. J. Almquist, H. Schmidt, P. Lang, J. Deitmer, M. Jirstrand, D. Prätzel-Wolters, H. Becker

Determination of interaction between MCT1 and CAII via a mathematical and physiological approach

Keywords: mathematical modeling; model reduction: electrophysiology; $\mathrm{pH}$-sensitive microelectrodes; proton antenna

(20 pages, 2008)
137. E. Savenkov, H. Andrä, O. Iliev

An analysis of one regularization approach for solution of pure Neumann problem Keywords: pure Neumann problem, elasticity, regularization, finite element method, condition number (27 pages, 2008)

138. O. Berman, J. Kalcsics, D. Krass, S. Nickel The ordered gradual covering location problem on a network

Keywords: gradual covering, ordered median function, network location

(32 pages, 2008)

139. S. Gelareh, S. Nickel

Multi-period public transport design: A novel model and solution approaches Keywords: Integer programming, hub location, public transport, multi-period planning, heuristics (31 pages, 2008)

140. T. Melo, S. Nickel, F. Saldanha-da-Gama Network design decisions in supply chain planning

Keywords: supply chain design, integer programming models, location models, heuristics

(20 pages, 2008)

141. C. Lautensack, A. Särkkä, J. Freitag K. Schladitz

Anisotropy analysis of pressed point processes

Keywords: estimation of compression, isotropy test nearest neighbour distance, orientation analysis, polar ice, Ripley's K function

(35 pages, 2008)

142. O. Iliev, R. Lazarov, J. Willems

A Graph-Laplacian approach for calculating the effective thermal conductivity of complicated fiber geometries

Keywords: graph laplacian, effective heat conductivity, numerical upscaling, fibrous materials

(14 pages, 2008)

143. J. Linn, T. Stephan, J. Carlsson, R. Bohlin Fast simulation of quasistatic rod deformations for VR applications

Keywords: quasistatic deformations, geometrically exact rod models, variational formulation, energy minimization, finite differences, nonlinear conjugate gradients

(7 pages, 2008)

\section{J. Linn, T. Stephan}

Simulation of quasistatic deformations using discrete rod models

Keywords: quasistatic deformations, geometrically exact rod models, variational formulation, energy minimization, finite differences, nonlinear conjugate gradients

(9 pages, 2008

145. J. Marburger, N. Marheineke, R. Pinnau Adjoint based optimal control using meshless discretizations

Keywords: Mesh-less methods, particle methods, Eulerian-Lagrangian formulation, optimization strategies, adjoint method, hyperbolic equations

(14 pages, 2008

\section{S. Desmettre, J. Gould, A. Szimayer}

Own-company stockholding and work effort preferences of an unconstrained executive Keywords: optimal portfolio choice, executive compensation

(33 pages, 2008) 
147. M. Berger, M. Schröder, K.-H. Küfer A constraint programming approach for the two-dimensional rectangular packing problem with orthogonal orientations

Keywords: rectangular packing, orthogonal orientations non-overlapping constraints, constraint propagation

(13 pages, 2008)

148. K. Schladitz, C. Redenbach, T. Sych, M. Godehardt

Microstructural characterisation of open foams using $3 d$ images

Keywords: virtual material design, image analysis, open foams

(30 pages, 2008)

149. E. Fernández, J. Kalcsics, S. Nickel, R. Ríos-Mercado

A novel territory design model arising in the implementation of the WEEE-Directive Keywords: heuristics, optimization, logistics, recycling (28 pages, 2008)

150. H. Lang, J. Linn

Lagrangian field theory in space-time for geometrically exact Cosserat rods Keywords: Cosserat rods, geometrically exact rods, small strain, large deformation, deformable bodies, Lagrangian field theory, variational calculus (19 pages, 2009)

151. K. Dreßler, M. Speckert, R. Müller, Ch. Weber

Customer loads correlation in truck engineering

Keywords: Customer distribution, safety critical components, quantile estimation, Monte-Carlo methods (11 pages, 2009)

152. H. Lang, K. Dreßler

An improved multiaxial stress-strain correction model for elastic FE postprocessing Keywords: Jiang's model of elastoplasticity, stress-strain correction, parameter identification, automatic differentiation, least-squares optimization, Coleman-Li algorithm

(6 pages, 2009)

153. J. Kalcsics, S. Nickel, M. Schröder

A generic geometric approach to territory design and districting

Keywords: Territory design, districting, combinatorial optimization, heuristics, computational geometry (32 pages, 2009)

154. Th. Fütterer, A. Klar, R. Wegener An energy conserving numerical scheme for the dynamics of hyperelastic rods

Keywords: Cosserat rod, hyperealstic, energy conservation, finite differences

(16 pages, 2009)

155. A. Wiegmann, L. Cheng, E. Glatt, O. Iliev, S. Rief

Design of pleated filters by computer simulations

Keywords: Solid-gas separation, solid-liquid separation, pleated filter, design, simulation

(21 pages, 2009)

156. A. Klar, N. Marheineke, R. Wegener Hierarchy of mathematical models for production processes of technical textiles
Keywords: Fiber-fluid interaction, slender-body theory, turbulence modeling, model reduction, stochastic differential equations, Fokker-Planck equation, asymptotic expansions, parameter identification

(21 pages, 2009)

157. E. Glatt, S. Rief, A. Wiegmann, M. Knefel, E. Wegenke

Structure and pressure drop of real and virtual metal wire meshes

Keywords: metal wire mesh, structure simulation, model calibration, CFD simulation, pressure loss (7 pages, 2009)

\section{S. Kruse, M. Mülle}

Pricing American call options under the assumption of stochastic dividends - An application of the Korn-Rogers model Keywords: option pricing, American options, dividends, dividend discount model, Black-Scholes model (22 pages, 2009)

159. H. Lang, J. Linn, M. Arnold

Multibody dynamics simulation of geometrically exact Cosserat rods

Keywords: flexible multibody dynamics, large deformations, finite rotations, constrained mechanical systems, structural dynamics

(20 pages, 2009)

160. P. Jung, S. Leyendecker, J. Linn, M. Ortiz Discrete Lagrangian mechanics and geometrically exact Cosserat rods

Keywords: special Cosserat rods, Lagrangian mechanics, Noether's theorem, discrete mechanics, frame-indifference, holonomic constraints

(14 pages, 2009)

161. M. Burger, K. Dreßler, A. Marquardt, M. Speckert

Calculating invariant loads for system simulation in vehicle engineering

Keywords: iterative learning control, optimal control

theory, differential algebraic equations(DAEs)

(18 pages, 2009)

162. M. Speckert, N. Ruf, K. Dreßler Undesired drift of multibody models excited by measured accelerations or forces Keywords: multibody simulation, full vehicle model, force-based simulation, drift due to noise (19 pages, 2009)

163. A. Streit, K. Dreßler, M. Speckert, J. Lichter, T. Zenner, P. Bach

Anwendung statistischer Methoden zur Erstellung von Nutzungsprofilen für die Auslegung von Mobilbaggern

Keywords: Nutzungsvielfalt, Kundenbeanspruchung, Bemessungsgrundlagen

(13 pages, 2009)

164. I. Correia, S. Nickel, F. Saldanha-da-Gama Anwendung statistischer Methoden zur Erstellung von Nutzungsprofilen für die Auslegung von Mobilbaggern

Keywords: Capacitated Hub Location, MIP formulations (10 pages, 2009)

165. F. Yaneva, T. Grebe, A. Scherrer

An alternative view on global radiotherapy optimization problems

Keywords: radiotherapy planning, path-connected sublevelsets, modified gradient projection method, improving and feasible directions

(14 pages, 2009)
166. J. I. Serna, M. Monz, K.-H. Küfer, C. Thieke Trade-off bounds and their effect in multicriteria IMRT planning

Keywords: trade-off bounds, multi-criteria optimization, IMRT, Pareto surface

(15 pages, 2009)

167. W. Arne, N. Marheineke, A. Meister, R. Wegener

Numerical analysis of Cosserat rod and string models for viscous jets in rotational spinning processes

Keywords: Rotational spinning process, curved viscous fibers, asymptotic Cosserat models, boundary value problem, existence of numerical solutions

(18 pages, 2009)

168. T. Melo, S. Nickel, F. Saldanha-da-Gama An LP-rounding heuristic to solve a multiperiod facility relocation problem

Keywords: supply chain design, heuristic, linear programming, rounding

(37 pages, 2009)

169. I. Correia, S. Nickel, F. Saldanha-da-Gama Single-allocation hub location problems with capacity choices

Keywords: hub location, capacity decisions, MILP formulations

(27 pages, 2009)

170. S. Acar, K. Natcheva-Acar A guide on the implementation of the Heath-Jarrow-Morton Two-Factor Gaussian Short Rate Model (HJM-G2++)

Keywords: short rate model, two factor Gaussian, G2++, option pricing, calibration

(30 pages, 2009)

171. A. Szimayer, G. Dimitroff, S. Lorenz A parsimonious multi-asset Heston model: calibration and derivative pricing Keywords: Heston model, multi-asset, option pricing, calibration, correlation

(28 pages, 2009)

172. N. Marheineke, R. Wegener Modeling and validation of a stochastic drag for fibers in turbulent flows Keywords: fiber-fluid interactions, long slender fibers, turbulence modelling, aerodynamic drag, dimensional analysis, data interpolation, stochastic partial differential algebraic equation, numerical simulations, experimental validations

(19 pages, 2009)

173. S. Nickel, M. Schröder, J. Steeg Planning for home health care services Keywords: home health care, route planning, metaheuristics, constraint programming

(23 pages, 2009)

174. G. Dimitroff, A. Szimayer, A. Wagner Quanto option pricing in the parsimonious Heston model

Keywords: Heston model, multi asset, quanto options, option pricing

(14 pages, 2009) 174. G. Dimitroff, A. Szimayer, A. Wagner

175. S. Herkt, K. Dreßler, R. Pinnau

Model reduction of nonlinear problems in structural mechanics

Keywords: flexible bodies, FEM, nonlinear model reduc tion, $P O D$

(13 pages, 2009) 
176. M. K. Ahmad, S. Didas, J. Iqbal

Using the Sharp Operator for edge detec-

\section{tion and nonlinear diffusion}

Keywords: maximal function, sharp function, image pro-

cessing, edge detection, nonlinear diffusion

(17 pages, 2009)

177. M. Speckert, N. Ruf, K. Dreßler, R. Müller, C. Weber, S. Weihe

Ein neuer Ansatz zur Ermittlung von Erprobungslasten für sicherheitsrelevante Bauteile

Keywords: sicherheitsrelevante Bauteile, Kundenbeanspruchung, Festigkeitsverteilung, Ausfallwahrscheinlichkeit, Konfidenz, statistische Unsicherheit, Sicherheitsfaktoren

(16 pages, 2009)

178. J. Jegorovs

Wave based method: new applicability

\section{areas}

Keywords: Elliptic boundary value problems, inhomogeneous Helmholtz type differential equations in bounded domains, numerical methods, wave based method, uniform $B$-splines

(10 pages, 2009)

179. H. Lang, M. Arnold

Numerical aspects in the dynamic simulation of geometrically exact rods

Keywords: Kirchhoff and Cosserat rods, geometrically exact rods, deformable bodies, multibody dynamics, artial differential algebraic equations, method of lines, time integration

(21 pages, 2009) 